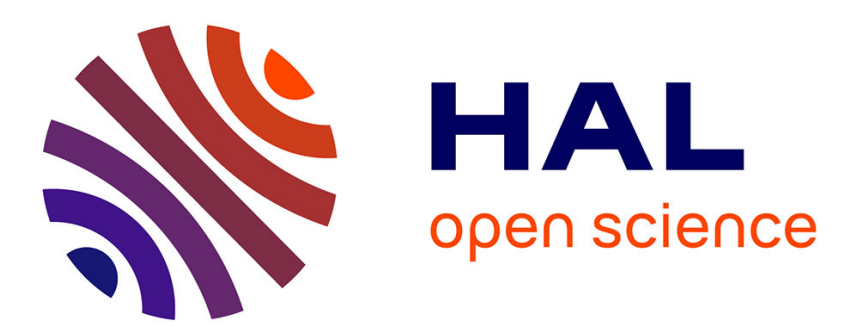

\title{
Simple and extensible plate and shell finite element models through automatic code generation tools
} Jack S Hale, Matteo Brunetti, Stéphane Bordas, Corrado Maurini

\section{To cite this version:}

Jack S Hale, Matteo Brunetti, Stéphane Bordas, Corrado Maurini. Simple and extensible plate and shell finite element models through automatic code generation tools. Computers \& Structures, 2018, 209, pp.163-181. hal-01763370v2

\section{HAL Id: hal-01763370 \\ https://hal.sorbonne-universite.fr/hal-01763370v2}

Submitted on 3 May 2018

HAL is a multi-disciplinary open access archive for the deposit and dissemination of scientific research documents, whether they are published or not. The documents may come from teaching and research institutions in France or abroad, or from public or private research centers.
L'archive ouverte pluridisciplinaire HAL, est destinée au dépôt et à la diffusion de documents scientifiques de niveau recherche, publiés ou non, émanant des établissements d'enseignement et de recherche français ou étrangers, des laboratoires publics ou privés. 


\title{
Simple and extensible plate and shell finite element models through automatic code generation tools
}

\author{
Jack S. Hale ${ }^{\mathrm{a}}$, Matteo Brunetti ${ }^{\mathrm{b}, \mathrm{c}}$, Stéphane P. A. Bordas ${ }^{\mathrm{a}, \mathrm{d}}$, Corrado Maurini ${ }^{\mathrm{b}, *}$ \\ ${ }^{a}$ Institute of Computational Engineering, University of Luxembourg, Luxembourg. \\ ${ }^{b}$ Dipartimento di Ingegneria Strutturale e Geotecnica, Sapienza Università di Roma, Italy. \\ ${ }^{c}$ Sorbonne Université, CNRS, Institut Jean Le Rond d'Alembert, UMR 7190, F-75005, Paris, France. \\ ${ }^{d}$ School of Engineering, Cardiff University, United Kingdom.
}

\begin{abstract}
A large number of advanced finite element shell formulations have been developed, but their adoption is hindered by complexities of transforming mathematical formulations into computer code. Furthermore, it is often not straightforward to adapt existing implementations to emerging frontier problems in thin structural mechanics including nonlinear material behaviour, complex microstructures, multi-physical couplings, or active materials. We show that by using a high-level mathematical modelling strategy and automatic code generation tools, a wide range of advanced plate and shell finite element models can be generated easily and efficiently, including: the linear and non-linear geometrically exact Naghdi shell models, the Marguerre-von Kármán shallow shell model, and the Reissner-Mindlin plate model. To solve shear and membrane-locking issues, we use: a novel re-interpretation of the Mixed Interpolation of Tensorial Component (MITC) procedure as a mixed-hybridisable finite element method, and a high polynomial order Partial Selective Reduced Integration (PSRI) method. The effectiveness of these approaches and the ease of writing solvers is illustrated through a large set of verification tests and demo codes, collected in an open-source library, FEniCS-Shells, that extends the FEniCS Project finite element problem solving environment.
\end{abstract}

Keywords: thin structures, plates, shells, finite element methods, domain specific language, FEniCS.

\section{Introduction}

Plates and shells are solids occupying a spatial domain with one dimension, the thickness, much smaller than the others. This implies the possibility of experiencing large changes of shape even with small material deformations and an approximately linear elastic material behaviour. Their study has received renewed attention in the last decade because of their unique nonlinear behaviour, mainly caused by geometric effects [10], leading to d-cone singularities and crumpling [30], fracture [79], or multistability $[84,85]$. Examples of modern application fields in engineering include shape control through active materials [48, 40], stretchable electronics [76], soft robotics [58], and thin nano-structures e.g. graphene sheets and nanotubes [9].

\footnotetext{
${ }^{*}$ Corresponding author

Email address: corrado.maurini@upmc.fr (Corrado Maurini)
} 
Effective models for plates and shells are two-dimensional, the through-the-thickness kinematics being described by including a suitable microstructure in the model. Their mathematical modelling leads to set of non-linear partial differential equations (PDEs) defined on a two-dimensional manifold embedded in three-dimensional space. The resulting PDEs can be discretised using a variety of numerical techniques, e.g. finite element methods (FEM) [13, 71], isogeometric analysis [45, 17], and meshfree methods [39]. FEM-based discretisations of the plate and shell models are available in both commercial (see e.g. Abaqus [2], ACEFem [51] and Adina [12] and Ls-Dyna) and open-source (e.g. GetFem ++ [72], IgaFem [70], Code_Aster [31], Mat-Fem [1] and Elmer [73]) software packages. However, formulating the mathematical models and implementing finite element solvers for custom plates and shells models remains a complex, highly technical, and time-consuming task, requiring advanced knowledge in differential geometry, numerical analysis, and mechanics. Shell and plate finite element models are regarded as very advanced topics in structural engineering and applied mathematics curricula, and rarely are graduate students trained in their implementation.

Our work aims to overcome some of the above difficulties by using modern automatic code generation tools and suitable mathematical abstractions. We present easily extensible and customisable methods for the implementation of finite element solvers for various models of thin structures, ranging from linear plates to non-linear shells. We leverage the tools offered by the finite element library FENICS PROJECT [4], in particular its Unified Form Language (UFL) [5] and the associated FENICS Form Compiler (FFC) [46], to allow users to straightforwardly formulate complex custom nonlinear shell models with less than one hundred lines of Python code and syntax that closely mirrors the mathematical abstractions of the variational formulation. We exploit the symbolic processing capabilities of UFL to specify the potential energy functionals of various thin structural models in a high-level way and select suitable locking-free finite element spaces for their discretisation. Custom constitutive models are straightforward to implement. Consistent linearisation of the potential energy functional is performed automatically using the symbolic differentiation tools in UFL [5]. These symbolic expressions are compiled automatically to finite element code using FFC [46]. The differential geometry concepts [28] are expressed directly in a very simple and expressive format, which we believe is of great pedagogical value. We mimic the geometrically-exact shell model [28], where the reference configuration is described by a mapping from $\mathbb{R}^{2}$ to a surface embedded in Euclidean space. Although we do not discuss here the implementation of specific preconditioners, our finite element solvers can run without modification on high-performance computing architectures using MPI and PETSC [11] allowing large-scale calculations to be performed. State-of-the-art linear and non-linear solution strategies are immediately available through PETSC [11]. These aspects sum up to a unique approach that we believe will be of value to researchers, students, and practitioners working on frontier problems in the mechanics of thin structures. The outcome of our work is distributed in the form of an open-source (LGPLv3) Python library, FEnICS-Shells [36], collecting an implementation of the models and discretisation techniques presented in this paper and including a large set of examples and documented demos.

We consider here several shearable plate and shell models, including linear plates (ReissnerMindlin model [75, 65]), linear and nonlinear shells (Nagdhi model $[67,66]$ ), and weakly non-linear shallow shells (Marguerre-von Kármán model [62]). For these structural models, the discretisation strategy is particularly important, because shear and membrane locking can lead to an unacceptably slow convergence rate of the finite element solution with the mesh size [25]. After a deep analysis of the methods available in the literature and the possible software tools, we selected two main 
discretisation techniques: the popular Mixed Interpolation of Tensorial Components (MITC) approach $[13,14,32]$ and a high polynomial-order Partial Selective Reduced Integration (PSRI) approach [7], both of which can be applied in a uniform manner across user-defined models.

Both the MITC and PSRI approches presented here include some original aspects with respect to the formulations previously introduced in the literature. In order to fit within the abstractions of UFL and the associated code generation tools, MITC is reformulated as a mixed hybridisable variational form with element-wise local projection. The PSRI technique proposed in [7] is extended to nonlinear shells with a modified reduced integration rule and optimised weighting factor for the energy splitting. To provide an efficient parallel implementation of the MITC technique, we also extended the FENICS automatic assembly tools to include the possibility of eliminating local degrees of freedom through static condensation, a feature that could be potentially ported to the FEnICS library itself in the near future.

The effectiveness of our MITC and PSRI discretisations are shown through a large set of numerical verification tests. Beside many classical examples, we propose a novel numerical verification test based on the exact solution of Mansfield [59] for the thermal buckling of a lenticular plate. We believe that this latter example can usefully complement the traditional gallery of verification tests for plates and shells.

The paper is organised as follows. First, we give an overview of the structural models considered in our work, presenting the research of their equilibrium configurations as an energy minimisation problem (Section 2). Hence we discuss the mathematics behind their finite element discretisation and some details about their practical implementation (Section 3). Finally we show some comprehensive verification examples demonstrating what is possible with our approach and the performance of the proposed methods (Section 4). Three detailed documented demos are included as supplementary material and several more are available in the online repository of the python library FENICSSHELls, companion to this paper.

A permanent DOI [36] has been created with links to the latest code and documentation. Table 1 summarises the mathematical models and the numerical methods presented in each of the documented demos currently available online. We suggest the reader consult the demos to have an overview of the main capabilities and features of FENICS-SHELLS, and in particular the Clamped Reissner-Mindlin plate under uniform load demo for linear plates, the Buckling of a heated von-Kármán plate for weakly nonlinear plate models, and the Clamped semi-cylindrical Naghdi shell under point load demo for fully nonlinear shells. FENICS-SHELLS follows the Best Practices for Scientific Computing [91] as closely as possible, including using version control, continuous integration and testing, repeatable computing environments [37] and providing thorough documentation.

\section{Structural models}

This section presents the variational formulation of the equilibrium problem for the basic plate and shell models considered in this work. Starting from the most general model, the nonlinear Naghdi shell model [66], the linear Reissner-Mindlin plate model [75, 65] is obtained as a special case of the linear Naghdi shell model [67]. In passing, we present also the so-called Marguerre-von Kármán shallow shell model [62], because (in its unshearable version) it is extensively used in the literature to study nonlinear phenomena in plates and shallow shells $[60,53,10]$. We review below the basic notations used in this work. 


\begin{tabular}{lll}
\hline Title & Model & Discretisation \\
\hline Clamped Reissner-Mindlin plate under uniform load & Linear shearable plate & MITC \\
Simply supported Reissner-Mindlin plate & Linear shearable plate & MITC \\
Clamped Reissner-Mindlin plate with MITC7 & Linear shearable plate & MITC \\
Clamped Kirchhoff-Love plate & Linear unshearable plate & DG \\
Buckling of a heated von Kármán plate & Shearable weakly nonlinear plate & PSRI \\
Non-linear Naghdi roll-up cantilever & Nonlinear shearable shell & MITC \\
Clamped semi-cylindrical Naghdi shell under point load & Nonlinear shearable shell & PSRI \\
Partly Clamped Hyperbolic Paraboloid & Linear shearable shell & PSRI \\
\hline
\end{tabular}

Table 1: Structural models and discretisation techniques presented in main documented demos provided in FENICSShElls [36]. All the demos adopt shareable shell theory, except the Clamped Kirchhoff-Love platewhich is based on the discontinuous Galerkin discretisation technique presented in [90, 69], not discussed in the present paper.

Notation. We equip the Euclidean space with a Cartesian frame $\left\{O ; a_{1}, \ldots, a_{n}\right\}$ with orthogonal unit basis vectors $a_{i} \in \mathcal{V}^{n}, \mathcal{V}^{n}$ being the translation space. Let $x=X-O \in \mathcal{V}^{n}$ denote the position vector of the point $X$ whose rectangular Cartesian coordinates are $\left(X_{1}, \ldots, X_{n}\right) \in \mathbb{R}^{n}$. We denote by $\mathbb{R}^{m \times n}$ the space of $m \times n$ matrices and by $\mathbb{S}^{n}=\left\{S \in \mathbb{R}^{n \times n}: S=S^{T}\right\}$ the space of symmetric $n \times n$ matrices, $\mathbb{S}_{+}^{n}$ its subset of positive definite matrices. With a notational abuse, for any tensor field $T \in \mathcal{V}^{n} \otimes \cdots \otimes \mathcal{V}^{n}$, let $T \in \mathbb{R}^{n \times \cdots \times n}$ denote also the collection of its Cartesian components. The same applies to vectors and tensors on manifolds. We denote by a the inner product between two tensors. In particular if $T$ is a tensor of order $2 n$ and $v$ a tensor of order $n$, we denote by $T v$ the application of the tensor $T$ on $v$ and by $T v \cdot v$ the quadratic form on the space of $v$ having $T$ as metric. For $v \in \mathbb{R}^{n}$ and $x \in \mathbb{R}^{m}, \nabla v \in \mathbb{R}^{n \times m}$ denotes the linear operator such that $\mathrm{d} v=\nabla v \mathrm{~d} x+o\left(|\mathrm{~d} x|^{2}\right)$. We often use also index notation, where Latin indices $(e . g . i, j)$ are assumed to take the values $1,2,3$, whilst Greek indices (e.g. $\alpha, \beta)$ take the values 1,2. For the partial derivatives of a function $f$ we use the abridged notation $f,{ }_{\alpha}=\partial f / \partial x_{\alpha}$. We define the space $L^{2}(\omega)$ as the usual Sobolev space of square-integrable functions on the domain $\omega$ with respect to the Lebesgue measure $\mathrm{d} x$. Then $H^{s}(\omega)$ is the usual Sobolev space of $L^{2}(\omega)$ functions whose weak derivatives of order $s \in \mathbb{N}$ are also in $L^{2}(\omega)$. We use the notation $f \in H^{s}\left(\omega ; \mathbb{R}^{n}\right)$ to denote a vector-valued function whose components $\left(f_{1}, \cdots, f_{n}\right)$ are each functions in $H^{s}(\omega)$.

\subsection{Non-linear Naghdi shell model}

Kinematics. We describe the reference configuration of shell-like bodies as:

$$
p_{0}\left(x_{\alpha}, x_{3}\right)=\phi_{0}\left(x_{\alpha}\right)+x_{3} n_{0}\left(x_{\alpha}\right), \quad x_{\alpha} \in \omega \subset \mathbb{R}^{2}, \quad x_{3} \in[-t / 2, t / 2],
$$

where $\left(x_{\alpha}, x_{3}\right)$ are curvilinear coordinates, $\phi_{0}: \omega \rightarrow \mathbb{R}^{3}$ is a (piece of) regular surface, and $n_{0}=\left(\partial_{1} \phi_{0} \times \partial_{2} \phi_{0}\right) /\left|\partial_{1} \phi_{0} \times \partial_{2} \phi_{0}\right|$ ( $\times$ refers to the vector cross-product in only this context) is a continuous unit vector field normal to the middle surface $\phi_{0}(\omega)$, Fig. 1.

The vectors $g_{0, \alpha}=\partial_{\alpha} \phi_{0}$ define a covariant basis on the tangent plane at each point of the reference configuration. The slenderness condition requires the thickness $t$ to be much smaller that the diameter of the middle surface $\omega$. The geometry of the middle surface is completely described by the metric and curvature tensors, $a_{0}$ and $b_{0}$ respectively, whose covariant components are computed as:

$$
a_{0}=\nabla \phi_{0}^{T} \nabla \phi_{0}, \quad b_{0}=-\frac{1}{2}\left(\nabla \phi_{0}^{T} \nabla n_{0}+\nabla n_{0}^{T} \nabla \phi_{0}\right), \quad a_{0} \in \mathbb{S}_{+}^{2}, b_{0} \in \mathbb{S}^{2},
$$




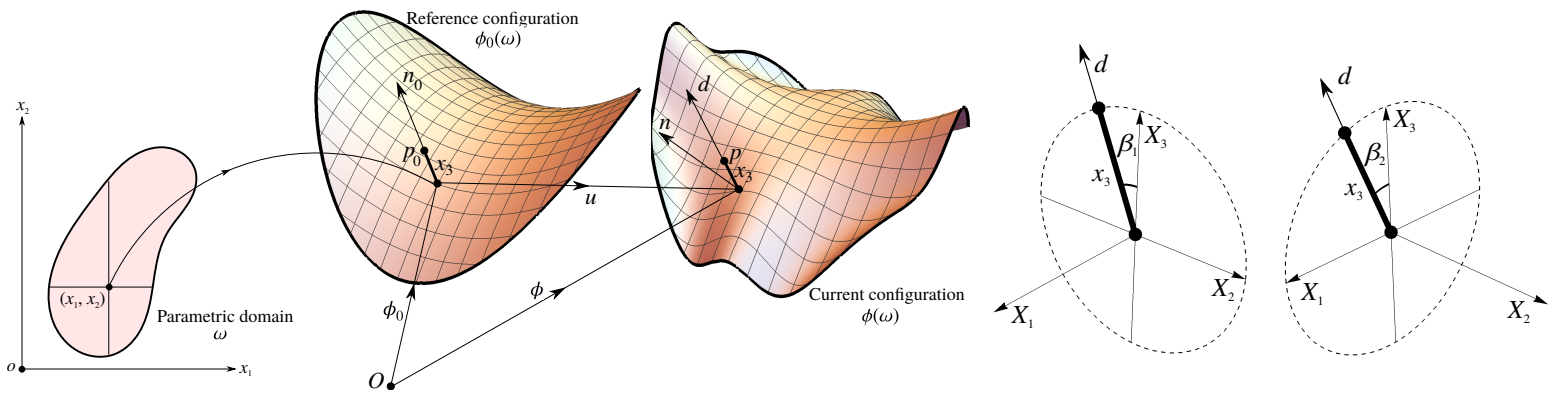

Figure 1: Kinematics of the nonlinear Naghdi shell model. Left: Parametric domain $\omega$ and placement of a generic point of the shell in the reference and current configuration. Right: Parameterisation of the director $d$ in terms of the $\beta_{1}, \beta_{2}$ angles.

where $\nabla \phi_{0}=\left[g_{0,1}, g_{0,2}\right]$. Whenever the reference configuration is chosen to be flat, that is when $\phi_{0}(\omega)$ is a planar surface, $n_{0} \equiv a_{3}$ (and $x_{3} \equiv X_{3}$ ) and $b_{0}=0$. In such a case a body is said plate-like.

In the nonlinear Naghdi shell model the current configuration of the shell is written as a sum of the placement of the middle surface $\phi: \omega \rightarrow \mathbb{R}^{3}$ (or alternatively the displacement field $u=\phi-\phi_{0}$ ) and of the director field $d: \omega \rightarrow \mathbb{R}^{3}$ (see Figure 1):

$$
p\left(x_{\alpha}, x_{3}\right)=\phi\left(x_{\alpha}\right)+x_{3} d\left(x_{\alpha}\right) .
$$

In general, $|d| \neq 1$ and $d \cdot n \neq 0$, where $n=\left(\partial_{1} \phi \times \partial_{2} \phi\right) /\left|\partial_{1} \phi \times \partial_{2} \phi\right|$ is the unit normal to the current surface and the vectors $g_{\alpha}=\partial_{\alpha} \phi$ define a covariant basis on the tangent plane at each point of the deformed configuration. When the normal fibres are assumed to be unstretchable, $|d|=1$ and the director can be parametrised by two angles $\beta=\left(\beta_{1}, \beta_{2}\right): \omega \rightarrow \mathbb{R}^{2}$, e.g., $d(\beta)=$ $\left(\cos \beta_{1} \sin \beta_{2},-\sin \beta_{1}, \cos \beta_{1} \cos \beta_{2}\right.$ ), giving rise the so-called five-parameter shell model [18] (the three-components of the displacement field and the two angles $\left.\left(\beta_{1}, \beta_{2}\right)\right)$, Figure 1 .

In a Lagrangian description, we use the following measures of deformation for the shell $[66,61]$ :

$$
\begin{aligned}
e_{N}(u) & =\frac{1}{2}\left(\nabla \phi(u)^{T} \nabla \phi(u)-a_{0}\right), \\
k_{N}(u, \beta) & =-\frac{1}{2}\left[\nabla \phi(u)^{T} \nabla d(\beta)+\nabla d(\beta)^{T} \nabla \phi(u)\right]-b_{0}, \\
\gamma_{N}(u, \beta) & =\nabla \phi(u)^{T} d(\beta),
\end{aligned}
$$

representing the stretching of the middle plane (a second order tensor), the bending curvature (a second order tensor), and the shear strain vector, respectively. The shear strain vanishes if and only if the director $d$ coincides with the unit normal to the surface.

Strain energy and constitutive equations. We consider elastic shells characterised by an elastic strain energy density in the reference configuration $\hat{\psi}(e, k, \gamma)$, a scalar function of the deformations. The stress measures are the membrane stress resultant $N=\partial_{e} \hat{\psi}$, a second order tensor, the couple stress resultant $M=\partial_{k} \hat{\psi}$ a second order tensor, and the vector $T=\partial_{\gamma} \hat{\psi}$ representing the transverse shear stress. We will focus in our examples on linearly elastic constitutive behaviour for which the strain energy density is a quadratic function of the strain measures. Thus, using standard notation:

$$
\hat{\psi}(e, k, \gamma)=\frac{1}{2} A(e-\bar{e}) \cdot(e-\bar{e})+B(e-\bar{e}) \cdot(k-\bar{k})+\frac{1}{2} D(k-\bar{k}) \cdot(k-\bar{k})+\frac{1}{2} S \gamma \cdot \gamma,
$$


where $A, D$ are fourth-order and positive-definite tensors providing the membrane and bending stiffness, $B$ is a fourth order tensor providing the membrane-to-bending constitutive coupling, $S$ is a second order tensor providing the shear stiffness and $\bar{e}, \bar{k}$ are symmetric second order tensor fields representing inelastic stretching and bending strains. In what follows, unless otherwise stated, $\bar{e}, \bar{k}=0$. This leads to the following linear constitutive laws:

$$
N=A(e-\bar{e})+B(k-\bar{k}), \quad M=B(e-\bar{e})+D(k-\bar{k}), \quad T=S \gamma .
$$

The stored energy density is the sum of membrane, $\hat{\psi}_{m}$, bending, $\hat{\psi}_{b}$, and shearing, $\hat{\psi}_{s}$, contributions:

$$
\hat{\psi}(e, k, \gamma)=\hat{\psi}_{m}+\hat{\psi}_{b}+\hat{\psi}_{s}=\frac{1}{2} N \cdot(e-\bar{e})+\frac{1}{2} M \cdot(k-\bar{k})+\frac{1}{2} T \cdot \gamma \cdot
$$

For a shell made of a single homogeneous layer of St. Venant-Kirchhoff isotropic material the contravariant components of the elastic tensors are:

$$
\frac{A^{\alpha \beta \sigma \tau}}{t}=12 \frac{D^{\alpha \beta \sigma \tau}}{t^{3}}=\frac{2 \lambda \mu}{\lambda+2 \mu} a_{0}^{\alpha \beta} a_{0}^{\sigma \tau}+\mu\left(a_{0}^{\alpha \sigma} a_{0}^{\beta \tau}+a_{0}^{\alpha \tau} a_{0}^{\beta \sigma}\right), \quad B^{\alpha \beta \sigma \tau}=0, \quad S^{\alpha \beta}=\mu a_{0}^{\alpha \beta},
$$

where $a_{0}^{\alpha \beta}$ are the contravariant components of the metric tensor $a_{0}$ and $\lambda, \mu$ the Lamé constants. Denoting by $\epsilon=t / L$ the small thinness parameter, $|A|,|S| \propto \epsilon$, while $|B| \propto \epsilon^{2}$ and $|D| \propto \epsilon^{3}$. Pure bending deformations $(e=0, \gamma=0)$ are energetically cheaper and, whenever possible, they are preferred.

Variational formulation of the equilibrium condition. We focus here on the solution of quasi-static problems under the action of conservative loads, although this is not a limitation of the FENICSSHELLS library. The equilibrium configurations are found by solving for the stationary points of the potential energy:

$$
\Pi_{N}(u, \beta)=\int_{\omega} \hat{\psi}\left(e_{N}(u), k_{N}(u, \beta), \gamma_{N}(u, \beta)\right) \sqrt{j_{0}} \mathrm{~d} x-W_{\text {ext }}
$$

where $W_{\text {ext }}$ is the external work of the conservative forces and $j_{0}=\operatorname{det} a_{0}$. Considering as Dirichlet boundary conditions imposed displacements $\bar{u}$ on the part of the boundary $\gamma_{u}$ and on the rotation $\bar{\beta}$ on $\gamma_{\beta}$, the space of admissible displacements and rotations are, respectively:

$$
\mathcal{U} \equiv\left\{u \in H^{1}\left(\omega, \mathbb{R}^{3}\right) \mid u=\bar{u} \text { on } \gamma_{u}\right\}, \quad \mathcal{R} \equiv\left\{\beta \in H^{1}\left(\omega, \mathbb{R}^{2}\right) \mid \beta=\bar{\beta} \text { on } \gamma_{\beta}\right\} .
$$

The variational formulation of the equilibrium equation consist in finding $(u, \beta) \in \mathcal{U} \times \mathcal{R}$ such that

$$
D_{\tilde{u}}\left[\Pi_{N}(u, \beta)\right]=0, \quad D_{\tilde{\beta}}\left[\Pi_{N}(u, \beta)\right]=0, \quad \forall(\tilde{u}, \tilde{\beta}) \in \mathcal{U}_{0} \times \mathcal{R}_{0},
$$

where $\mathcal{U}_{0}$ and $\mathcal{R}_{0}$ are the vector spaces associated to $\mathcal{U}$ and $\mathcal{R}$, obtained by setting $\bar{u}=0$ and $\bar{\beta}=0$ in (3). The stability of an equilibrium can by assessed by studying the sign of the second derivative of the energy, a quadratic form of the variations $\tilde{u}, \tilde{\beta}$.

Remark. Shell models with energy in the form (2) are shearable, since flexure is due to both bending and shearing. However, for very thin shells and plates the shearing strains turn out to be negligible. These structures are quite naturally modelled by means of unshearable or pure bending theories obtained by imposing the inner constraint $\gamma=0$. Such theories can usually be recovered 
as limit models of shearable theories when the thinness parameter goes to zero, $\epsilon \rightarrow 0$. In what follows the shell models in the library are introduced in their 'shearable version'; their unshearable counterpart can be easily derived. We remark that the distinction between shearable and unshearable theories is of paramount importance, since the numerical solution procedures exhibit quite different challenges.

Remark. By assuming the inner constraint $\gamma_{N}=0$, from Eq. $(1)_{3} d=n \in \operatorname{ker} \nabla \phi^{T}(u)$. Moreover, from Eq. (1) $)_{2}$, the change of curvature can be written as $k(u)=b(u)-b_{0}, b(u)$ being the curvature tensor of the current surface. In such a way the nonlinear Koiter shell model [49] is recovered as the unshearable counterpart of the Naghdi's one for thin shells. Since $b_{\alpha \beta}=n_{i, \beta}\left(\phi_{0}+u\right),_{\alpha}=$ $n_{i}\left(\phi_{0}+u\right)_{\alpha \beta},(i=1,2,3)$, the displacement $u$ must be sought in a space $\mathcal{U} \subseteq H^{2}\left(\omega, \mathbb{R}^{3}\right)$. This high regularity requirement is a common feature of the unshearable theories.

\subsection{Marguerre-von Kármán shallow shell model}

Some widely studied and used weakly non-linear versions of the Naghdi shell equations are the Föppl-von Kármán plate model [34, 89] and the Marguerre shallow shell model [62]. They can be seen as the simplest nonlinear models able to properly describe the moderately large deflections of thin shallow shells or plates, since they retain a minimal geometrical coupling between the membrane and flexural behaviour. Here, we introduce them in their shearable version [44].

Consider a shell with a shallow initial configuration $\phi_{0}\left(x_{\alpha}\right)=x_{\alpha} a_{\alpha}+\zeta\left(x_{\alpha}\right) a_{3}$, with the shallowness assumption $|\nabla \zeta| \sim \eta \ll 1$ and let $\phi\left(x_{\alpha}\right)=x_{\alpha} a_{\alpha}+v\left(x_{\alpha}\right)+w\left(x_{\alpha}\right) a_{3}$ be the current configuration, where $e_{3}$ of the middle plane with respect to a flat configuration $x=x_{\alpha} e_{\alpha} \in \omega$, and decompose the $v \perp \omega$ is the in-plane component and $w$ on the $a_{1}-a_{2}$ plane, with $v \cdot a_{3}=0$, and the transverse component $w a_{3}$. Similarly, we denote by $\theta$ the (small) rotations of the fibres orthogonal to the middle-plane, with $\theta \cdot a_{3}=0$.

The displacement of an arbitrary point of the three-dimensional body can be represented as:

$$
U_{M}\left(x_{\alpha}, x_{3}\right)=v\left(x_{\alpha}\right)+w\left(x_{\alpha}\right) a_{3}-x_{3} \theta\left(x_{\alpha}\right) .
$$

The Marguerre-von Kármán shallow shell model can be derived from the nonlinear Naghdi model by assuming $|v| / L \sim \eta^{2},|w| / L \sim \eta$ and $|\theta| \sim \eta$, so that $d\left(x_{\alpha}\right)-n_{0}\left(x_{\alpha}\right)=\theta\left(x_{\alpha}\right)+$ h.o.t., where $\theta\left(x_{\alpha}\right)=\beta\left(x_{\alpha}\right) \times a_{3}$. Then, the Marguerre-von Kármán strain measures are obtained as the leading order terms of the Naghdi strain measures, Eqs. 1,

$$
\begin{aligned}
e_{M}(v, w) & =\frac{\nabla v+\nabla v^{T}}{2}+\frac{\nabla w \otimes \nabla w}{2}-\frac{\nabla \zeta \otimes \nabla \zeta}{2}, \\
k_{M}(\theta) & =\frac{\nabla \theta+\nabla \theta^{T}}{2}-\nabla \nabla \zeta, \quad \gamma_{M}(w, \theta)=\nabla w-\theta .
\end{aligned}
$$

Then, solutions are found as the minimisers over a smooth set of vector fields $v \in \mathcal{V} \subseteq H^{1}\left(\omega, \mathbb{R}^{2}\right)$, $w \in \mathcal{W} \subseteq H^{1}(\omega), \theta \in \mathcal{R} \subseteq H^{1}\left(\omega, \mathbb{R}^{2}\right)$ satisfying appropriate boundary conditions, of the nonlinear Marguerre-von Kármán energy functional:

$$
\begin{aligned}
\Pi_{M}(v, w, \theta)=\frac{1}{2} \int_{\omega} N_{M}(v, w) \cdot\left(e_{M}(v, w)-\bar{e}\right) \mathrm{d} x+ & \frac{1}{2} \int_{\omega} M_{M}(w, \theta) \cdot\left(k_{M}(w, \theta)-\bar{k}\right) \mathrm{d} x \\
& +\frac{1}{2} \int_{\omega} T_{M}(w, \theta) \cdot \gamma_{M}(w, \theta) \mathrm{d} x-W_{\text {ext }} .
\end{aligned}
$$


Remark. By assuming the inner constraint $\gamma_{M}=0$, from Eq. (6) ${ }_{3}, \theta=\nabla w$. Moreover, from Eq. $(6)_{2}, k(w)=\nabla \nabla w$. In such a way the classical (unshearable) Marguerre-von Kármán shallow shell model is recovered. As for the Koiter model [49], the transverse displacement $w$ must be sought in a space $\mathcal{W} \subseteq H^{2}(\omega)$.

\subsection{Linear Naghdi shell model}

Consider a shell body with reference surface $\phi_{0}(\omega)$. Again, let $U_{\tilde{N}}\left(x_{\alpha}, x_{3}\right)=u\left(x_{\alpha}\right) a_{3}-x_{3} \theta\left(x_{\alpha}\right)$ be the representation formula for the displacement vector field, with $\theta \in T_{P} \phi_{0}\left(x_{\alpha}\right), T_{P} \phi_{0}\left(x_{\alpha}\right)$ being the tangent plane to the reference surface at the point $\phi_{0}\left(x_{\alpha}\right) ; u$ represents an (infinitesimal) displacement from the reference surface, $\theta$ denotes the (infinitesimal) rotation of the fibers orthogonal to the middle surface. The linear Naghdi strain measures are then obtained by linearisation from Eqs. (1),

$$
\begin{aligned}
e_{\tilde{N}}(u) & =\frac{1}{2}\left(\nabla \phi_{0}^{T} \nabla u+\nabla u^{T} \nabla \phi_{0}\right), \\
k_{\tilde{N}}(u, \theta) & =-\frac{1}{2}\left(\nabla \phi_{0}^{T} \nabla \theta+\nabla \theta^{T} \nabla \phi_{0}\right)-\frac{1}{2}\left(\nabla n_{0}^{T} \nabla u+\nabla u^{T} \nabla n_{0}\right), \\
\gamma_{\tilde{N}}(u, \theta) & =\nabla \phi_{0}^{T} \theta+\nabla u^{T} n_{0} .
\end{aligned}
$$

Then, solutions are found as the minimisers over a smooth set of vector fields $u \in \mathcal{U} \subseteq H^{1}\left(\omega, \mathbb{R}^{3}\right)$, $\theta \in \mathcal{R} \subseteq H^{1}\left(\omega, \mathbb{R}^{2}\right)$ satisfying appropriate boundary conditions, of the linear Naghdi energy functional:

$$
\begin{aligned}
\Pi_{\tilde{N}}(u, \theta)=\frac{1}{2} \int_{\omega} N_{\tilde{N}}(u) \cdot e_{\tilde{N}}(u) \mathrm{d} x+\frac{1}{2} \int_{\omega} M_{\tilde{N}}(u, \theta) \cdot k_{\tilde{N}}(u, \theta) \mathrm{d} x & \\
& +\frac{1}{2} \int_{\omega} T_{\tilde{N}}(u, \theta) \cdot \gamma_{\tilde{N}}(u, \theta) \mathrm{d} x-W_{\text {ext }} .
\end{aligned}
$$

Remark. For a plate-like body, Eqs. (8) reduce to:

$$
e_{R M}(v)=\frac{1}{2}\left(\nabla v+\nabla v^{T}\right), \quad k_{R M}(v, \theta)=\frac{1}{2}\left(\nabla \theta+\nabla \theta^{T}\right), \quad \gamma_{R M}(w, \theta)=\nabla w-\theta,
$$

where $u\left(x_{\alpha}\right)=v\left(x_{\alpha}\right)+w\left(x_{\alpha}\right) a_{3}$. In such a case the membrane and flexural problem are geometrically decoupled and a linear coupling turns out to be possible only at the constitutive level. Eqs. (10) 2,3 are the Reissner-Mindlin plate strain measures, depending only by the transverse displacement $w$ and the rotation $\theta$. Again, solutions are found as the minimisers over a smooth set of vector fields $w \in \mathcal{W} \subseteq H^{1}(\omega), \theta \in \mathcal{R} \subseteq H^{1}\left(\omega, \mathbb{R}^{2}\right)$, satisfying appropriate boundary conditions, of the Reissner-Mindlin energy functional:

$$
\Pi_{R M}(w, \theta)=\frac{1}{2} \int_{\omega} M_{R M}(w, \theta) \cdot k_{R M}(w, \theta) \mathrm{d} x+\frac{1}{2} \int_{\omega} T_{R M}(w, \theta) \cdot \gamma_{R M}(w, \theta) \mathrm{d} x-W_{e x t} .
$$

Remark. By assuming the inner constraints $\gamma_{\tilde{N}}=0$ and $\gamma_{R M}=0$, the linear Koiter shell model [50] and the Kirchhoff-Love [47, 57] plate model are recovered, respectively. Solutions of such unshearable models must be sought in spaces $\mathcal{U} \subseteq H^{2}\left(\omega, \mathbb{R}^{3}\right), \mathcal{W} \subseteq H^{2}(\omega)$, respectively. 


\section{Finite element formulations and implementation details}

Notation. We use nomenclature from [55] related to finite element methods. We let $\mathcal{T}_{h}$ (the mesh) be a partition of the domain $\omega$ into a finite set of triangular cells $\mathcal{T}_{h}=\{T\}$ with disjoint interiors:

$$
\cup_{T \in \mathcal{T}_{h}} T=\omega .
$$

Each triangular cell $T$ has circumradius $h_{T}$, and $h:=\max _{T \in \mathcal{T}_{h}} h_{T}$. We then denote the set of edges of $T_{h}$ as $\mathcal{E}=\{E\} . \mathcal{E}_{h}^{\text {int }} \subset \mathcal{E}_{h}$ is the subset of edges on the interior of the mesh (i.e. not on the boundary). Normals to the edges of each cell are denoted $n$. Functions $u$ evaluated on opposite sides of an edge $E$ are indicated by the subscripts + and - , giving the jump operator $\llbracket u \rrbracket=u_{+} \cdot n_{+}+u_{-} \cdot n_{-}$. We denote $C G_{p} \subset H^{1}(\omega)$ the standard continuous Lagrangian finite element space of polynomial order $p$ constructed on the mesh $\mathcal{T}_{h}$. The notation $f \in\left[C G_{p}\right]^{n} \subset H^{s}\left(\omega ; \mathbb{R}^{n}\right)$ denotes a vector-valued function whose components $\left(f_{1}, \cdots, f_{n}\right)$ are each a member of $C G_{p}$.

\subsection{Background}

As pointed out in the previous section, shearable models give rise to weak formulations with solutions in $H^{1}$ while unshearable models give rise to weak formulations with solutions in $H^{2}$. It is well-known that producing conforming finite element method discretisations of $H^{2}$ is not straightforward, although several elegant solutions are possible $[29,69,17,39]$. To bypass this issue, one can choose to use shearable models even for very thin structures, even though in such cases shear strains are negligible. Shearable models only requires $H^{1}$-conforming finite element methods which are significantly easier to implement than either discontinuous Galerkin or $H^{2}$-conforming finite element methods.

Unfortunately it is also the case that applying standard $H^{1}$ conforming finite element methods leads to shear-locking in the thin limit, since the finite element spaces are unable to properly represent the zero shear-strain $\gamma=0$ constraint asymptotically enforced within the mathematical model as the thinness parameter goes to zero. A similar problem arises in shell problems whenever pure bending displacements are non-inhibited: $H^{1}$ conforming finite element methods for such bending-dominated shells suffer of membrane-locking [25, 81, 82]. In other words, the discrete spaces are incompatible with the structural models.

A wide variety of locking-free numerical methods exist, including the methods of assumed natural strains [14, 26, 43], enhanced assumed strains [86, 19], discrete shear gap [20], partial selective reduced integration [7] and mixed variational [39] approaches have been developed to overcome this problem. Implementing these locking-free approaches is more complex than implementing the standard $H^{1}$-conforming finite element method as they require mixed formulations and special finite element function spaces. Making these methods easier to code, use and adapt is one of the main goals of the present work and the companion open-source library FENICS-SHELLS.

For alleviating shear-locking in the Reissner-Mindlin and Marguerre-von Kármán models we have chosen to implement a Mixed Interpolation Tensorial Components (MITC) reduction operator. The MITC approach was introduced in the paper of Bathe and Dvorkin [13] for curing shear-locking in the finite element discretisation of the Reissner-Mindlin model. Other notable contributions along the same lines include the Durán-Liberman [32] and the MITC7 and MITC9 [14] elements. The success of the various MITC elements for the Reissner-Mindlin problem rests on solid results in numerical analysis, e.g. [14, 3]. It has also been shown numerically that MITC approach can be successful in solving membrane and shear locking issues in the linear and non-linear Naghdi shell 
models [43], although without rigorous mathematical justification. A fully general shell element with guaranteed mathematical and numerical behaviour in membrane and bending-dominated regimes is still out of reach and mathematically robust finite element design for shells is an active research topic. We have chosen to implement a high-order partial selective reduced integration (PSRI) method which was analysed for the linear Naghdi model in [7].

\subsection{The shear and membrane-locking issues}

For notational convenience we drop the explicit RM suffix relating to the Reissner-Mindlin model in what follows. Informally, when considering the Reissner-Mindlin plate model, shear-locking is the result of the Kirchhoff constraint (vanishing shear strain $\gamma$ ) [38]:

$$
\gamma=\nabla w-\theta=0,
$$

being enforced on the discrete finite element spaces $w_{h} \in \mathcal{W}_{h} \subset \mathcal{W}$ and $\theta_{h} \in \mathcal{R}_{h} \subset \mathcal{R}$ for as the small thinness parameter $\epsilon \rightarrow 0$. The approximation power in the discrete finite element basis is inadequate to simultaneously represent the Kirchhoff (bending) dominated mode and also provide a uniformly convergent approximation to the problem with respect to $\epsilon$.

More formally, denoting the continuous space of pure bending displacements $(\epsilon=0)$ as:

$$
\mathcal{V}_{0}=\{(w, \theta) \in \mathcal{W} \times \mathcal{R} \mid \nabla w-\theta=0\},
$$

and the discrete solution $\left(w_{h}, \theta_{h}\right) \in \mathcal{V}_{h}:=\mathcal{W}_{h} \times \mathcal{R}_{h}$, we seek solutions in a subspace $\mathcal{V}_{h 0} \subset\left(\mathcal{V}_{h} \cap \mathcal{V}_{0}\right)$ containing discrete pure bending displacements:

$$
\mathcal{V}_{h 0}=\left\{\left(w_{h}, \theta_{h}\right) \in \mathcal{W}_{h} \times \mathcal{R}_{h} \mid \nabla w_{h}-\theta_{h}=0\right\} .
$$

It is not so hard to construct discretisations in which this requirement is quite catastrophic. Consider using standard $H^{1}(\omega)$-conforming linear Lagrangian elements for both fields, $\mathcal{W}_{h}:=\mathrm{CG}_{1}$ and $\mathcal{R}_{h}:=\left[\mathrm{CG}_{1}\right]^{2}$, and suppose fully clamped Dirichlet conditions on all of the boundary, that is $\mathcal{W}_{h} \subset H_{0}^{1}(\omega)$ and $\mathcal{R}_{h} \subset H_{0}^{1}\left(\omega, \mathbb{R}^{2}\right)$. With piecewise linear functions for $w_{h}, \nabla w_{h}$ then consists of piecewise constant functions. Furthermore, as $\theta_{h}$ must be zero on the boundary, and we are searching for solutions in the space $\mathcal{V}_{h 0}$ where $\nabla w_{h}=\theta_{h}$, the only possible solution that our discrete space can represent is $w_{h}=0$ and $\theta_{h}=0$. The finite element model is overly stiff, hence the term 'locking'.

The membrane-locking issue arises in the linear and non-linear Naghdi shell models and the Marguerre-von Kármán shallow shell model. The locking issue can be motivated by a similar argument to the shear-locking issue and causes similarly poor results. However, the precise circumstances under which membrane-locking can appear is a more subtle matter, and can depend on a combination of geometry, boundary conditions and loading. We refer the reader to [25] for an excellent discussion of locking in shell finite element models. Here, we limit ourselves to remarks we consider strictly necessary to make our exposé understandable.

For simplicity in what follows, we refer to the linear Naghdi shell model. In this case, the continuous space of pure bending displacements (no membrane or shear strains) reads as:

$$
\mathcal{V}_{0}=\left\{(u, \theta) \in \mathcal{U} \times \mathcal{R} \mid e_{N}(u)=0 \text { and } \gamma_{N}(u, \theta)=0\right\} .
$$

When the thinness parameter goes to zero two distinct asymptotic behaviours emerge, depending on whether $\mathcal{V}_{0}$ is empty or not, and thus on the shape of the shell and the Dirichlet boundary 
conditions applied on it. If $\mathcal{V}_{0}=\emptyset$, pure bending is inhibited and the shell carries the load mainly by membrane actions; this is the so called membrane-dominated regime. Conversely, whenever $\mathcal{V}_{0} \neq \emptyset$, pure bending is non-inhibited, the membrane and shearing contribution to the elastic energy vanish and the shell carries the load by bending actions; this is the so called bending-dominated regime. When considering finite element discretisations, this latter case is likely to suffer both membrane and shear locking, unless the discrete space $\mathcal{V}_{h 0}$ is chosen so as to properly approximate the space $\mathcal{V}_{0}$ of pure bending displacements. In any case, since a finite element formulation should perform well regardless of the asymptotic regime, benchmark tests have to be provided for both membraneand bending-dominated shells.

\subsection{Mixed Interpolation of Tensorial Components (MITC) method}

We use the MITC approach to deal with the shear-locking issue. The MITC approach is based on an underlying mixed variational method, where the Reissner-Mindlin shear strains $\gamma$ are considered as an independent variable. One of the most attractive aspects of the MITC approach is that the final linear system of equations only contains the original primal unknown fields $\left(w_{h}, \theta_{h}\right)$ despite the consideration of the shear strain field $\gamma_{h}$ in the formulation.

The first step in constructing an MITC-type element is the correct identification of the function space $\mathcal{S}\left(\omega ; \mathbb{R}^{2}\right)$ in which the shear strain $\gamma$ lives. For any scalar field $w \in H^{1}(\omega)$ it holds that $\nabla w \in H(\operatorname{rot} ; \omega)$ where $H(\operatorname{rot} ; \omega)$ is the Sobolev space of square-integrable vector-valued functions with square-integrable rot:

$$
H(\operatorname{rot} ; \omega):=\left\{\gamma \in L^{2}\left(\omega ; \mathbb{R}^{2}\right) \mid \operatorname{rot} \gamma:=\partial_{1} \gamma_{2}-\partial_{2} \gamma_{1} \in L^{2}(\omega)\right\} .
$$

Furthermore, for $\theta \in H^{1}\left(\omega ; \mathbb{R}^{2}\right)$ we have $\operatorname{rot} \theta \in L^{2}(\omega)$ and thus it follows that

$$
\gamma=\nabla w-\theta \in \mathcal{S} \equiv H(\operatorname{rot} ; \omega) .
$$

Note that the operator rot is synonymous with the operator curl in $\mathbb{R}^{2}$.

Finite element approximation spaces that satisfy $\mathcal{S}_{h} \subset \mathcal{S}$ consist of the well-known 'curl/rotconforming' elements, namely the Nédélec [68] (or rotated Raviart-Thomas elements [74] in $\mathbb{R}^{2}$ ) of the first $\mathrm{NED}_{1}^{n}$ and second kind $\mathrm{NED}_{2}^{n}$, and the Brezzi-Douglas-Fortin-Marini $\left(\mathrm{BDFM}^{n}\right)$ elements [23], where $n$ is the element (polynomial) order. All of these finite element spaces are available for use in FENICS $[4,54,5]$. We refer the reader to [55] for a full description of the mathematical construction of these elements, and [77] as a reference for the issues surrounding their implementation in FENICS.

For brevity, we shown only the development of the Durán-Liberman element [32]. This element can be considered the lowest order MITC-type element with rigorous analytical convergence proofs. There is also an implementation of MITC7 [14] in FEnICS-SHELLS but do not show the development in this paper.

The starting point is the discrete counterpart of eq. (11):

$$
\begin{gathered}
\Pi\left(w_{h}, \theta_{h}\right)=\Pi^{b}\left(w_{h}, \theta_{h}\right)+\frac{1}{2} \int_{\omega} T\left(w_{h}, \theta_{h}\right) \cdot \gamma\left(w_{h}, \theta_{h}\right) \mathrm{d} x-W_{\text {ext }}, \\
\Pi^{b}\left(w_{h}, \theta_{h}\right):=\frac{1}{2} \int_{\omega} M\left(w_{h}, \theta_{h}\right) \cdot k\left(w_{h}, \theta_{h}\right) \mathrm{d} x .
\end{gathered}
$$

The finite element spaces used in the construction of the Durán-Liberman element are continuous piecewise-linear Lagrangian elements for the transverse displacements $w_{h} \in \mathcal{W}_{h}:=\mathrm{CG}_{1}$, continuous 


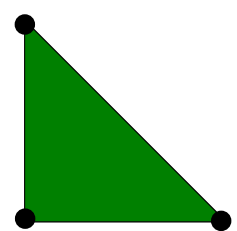

$w_{h} \in \mathrm{CG}_{1}$

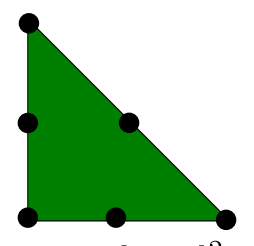

$\theta_{h} \in\left[\mathrm{CG}_{2}\right]^{2}$

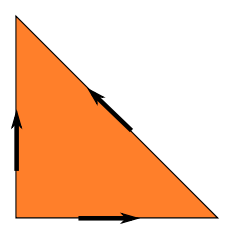

$\gamma_{h}, p_{h} \in \mathrm{NED}_{1}$

Figure 2: Finite element spaces making up the Durán-Liberman element. Circles denote point evaluations for degrees of freedom. Arrows denote point evaluations of tangential component for degrees of freedom. The Durán-Liberman has a total of 21 degrees of freedom: 3 for the displacement $w_{h}, 12$ for the rotations $\theta_{h}, 3$ for the shear strain $\gamma_{h}$, and 3 for the Lagrange multiplier $p_{h}$. Thanks to the static condensation at the cell level, the latter 6 degrees of freedom for $\gamma_{h}$ and $p_{h}$ can be eliminated during the assembling process, reducing the number of effective degree of freedom per element to 15 .

piecewise-quadratic vector-valued Lagrangian elements for the rotations $\theta_{h} \in \mathcal{R}_{h}:=\left[\mathrm{CG}_{2}\right]^{2}$ and, finally, the lowest-order Nédélec elements of the first kind for the shear strain $\gamma_{h} \in \mathcal{S}:=\mathrm{NED}_{1}{ }^{1}$. The product of these spaces then makes up the Durán-Liberman element, see Figure 2.

The element $\mathrm{NED}_{1}$ is defined [55] by the Ciarlet triple $(T, \mathcal{V}, \mathcal{L})$ (cell, polynomial space, degrees of freedom):

$$
\begin{aligned}
& T=\text { triangle, } \\
& \mathcal{V}=\left[\mathcal{P}_{0}(T)\right]^{2}+\mathcal{S}_{1}(T), \\
& \mathcal{L}=\int_{E} v \cdot t p \mathrm{~d} s, \text { for } p \in \mathcal{P}_{0}(E), \forall E \subset T,
\end{aligned}
$$

where $T$ is the reference triangle with edges $E$ with measure $\mathrm{d} s, \mathcal{P}_{0}$ is the set of constant polynomial functions on the triangle $T$ or edge $E$, and $\mathcal{S}_{1}(T)$ is the polynomial space:

$$
\mathcal{S}_{1}(T)=\left\{s \in\left[\mathcal{P}_{1}(T)\right]^{2} \mid s(x) \cdot x=0 \quad \forall x \in T\right\} .
$$

The 'trick' of the MITC approach is recognising that by modifying the shear strain field at the discrete level by applying a special operator $R_{h}$ that takes the primal to the conforming space $H($ rot; $\omega)$ for the shear strains

$$
R_{h}:\left[\mathrm{CG}_{1} \times\left[\mathrm{CG}_{2}\right]^{2}\right] \rightarrow \mathrm{NED}_{1}
$$

the element can be 'unlocked'. For the Durán-Liberman element, the operator $R_{h}$ can be defined by the following condition for each cell $T$ in the mesh $\mathcal{T}_{h}$ :

$$
\int_{E}\left[\left(R_{h}\left[\gamma\left(w_{h}, \theta_{h}\right)\right]-\gamma_{h}\right) \cdot t\right] p \mathrm{~d} s=0, \forall p \in \mathcal{P}_{0}(E), \forall E .
$$

\footnotetext{
${ }^{1}$ The original element construction of Durán and Liberman uses a slightly smaller quadratic space constructed as a sum of linear polynomials and quadratic bubbles. As this space is not available in FENICS we use the full quadratic space instead.
} 
Note that (22) is very similar to the degree of freedom definition $\mathcal{L}$ in (19c). In effect, $R_{h}$ 'ties' the degrees of freedom of the transverse displacement and rotation space $C G_{1} \times\left[C G_{2}\right]^{2}$ to an underlying $\mathrm{NED}_{1}$ space. Then, applying the operator $R_{h}$ to (18) gives:

$$
\Pi\left(w_{h}, \theta_{h}\right)=\Pi^{b}\left(w_{h}, \theta_{h}\right)+\frac{1}{2} \int_{\omega} T\left(R_{h} \gamma\left(w_{h}, \theta_{h}\right)\right) \cdot R_{h} \gamma\left(w_{h}, \theta_{h}\right) \mathrm{d} x-W_{\text {ext }} .
$$

Remark. For the Reissner-Mindlin strain measure $R_{h}\left[\gamma\left(w_{h}, \theta_{h}\right)\right]=\nabla w_{h}-R_{h} \theta_{h}$ because $R_{h} w_{h}=$ $w_{h}$ by definition. This application of the reduction operator to the rotation field is often found in the literature. However, in our work we always apply the reduction operator to the complete shear-strain variable $\gamma$.

\subsection{New implementation of the MITC method in FENICS-SHELLS}

The formulation and implementation of MITC in FENICS-ShELLS is slightly different to the standard approach. In our view it is simpler to understand as it is purely variational and extends the MITC shear-locking cure quite naturally to the non-linear models. Another key advantage is that it can be concisely expressed in UFL [5].

Starting with (18) and this time directly substituting in the conforming strain field $\gamma_{h} \in \mathcal{S}_{h}$ gives:

$$
\Pi\left(w_{h}, \theta_{h}, \gamma_{h}\right)=\Pi^{b}\left(w_{h}, \theta_{h}\right)+\int_{\omega} T\left(\gamma_{h}\right) \cdot \gamma_{h} \mathrm{~d} x-W_{\text {ext }} .
$$

We then 'tie' the tangential component of the conforming strain field $\gamma_{h} \cdot t$ to its counterpart calculated using the primal unknowns $\gamma \cdot t \equiv(\nabla w-\theta) \cdot t$ using a Lagrange multiplier field $p_{h} \in \mathrm{NED}_{1}$ that lives on all edges $E \in \mathcal{E}_{h}$ of the mesh $\mathcal{T}_{h}$ :

$$
\Pi\left(w_{h}, \theta_{h}, \gamma_{h}, p_{h}\right)=\Pi^{b}\left(w_{h}, \theta_{h}\right)+\int_{\omega} T\left(\gamma_{h}\right) \cdot \gamma_{h} \mathrm{~d} x+\sum_{E \in \mathcal{E}_{h}} \int_{E}\left(\gamma\left(w_{h}, \theta_{h}\right) \cdot t-\gamma_{h} \cdot t\right) \cdot\left(p_{h} \cdot t\right) \mathrm{d} s-W_{\text {ext }} .
$$

It is possible to implement the Durán-Liberman element in FENICS by directly expressing (25) using UFL. However, this comes at the expense of two extra fields $\gamma_{h}$ and $p_{h}$ over the standard MITC implementation.

Fortunately it is possible to eliminate both $\gamma_{h}$ and $p_{h}$ at the cell-local level. To this end, we begin by defining the residual $F(q ; \tilde{q})=0$ as the usual Gâteaux derivative of $(25)$ at a point $q_{h}:=\left(w_{h}, \theta_{h}, \gamma_{h}, p_{h}\right)$ in the direction of test functions $\tilde{q}:=(\tilde{w}, \tilde{\theta}, \tilde{\gamma}, \tilde{p})$ :

$$
F\left(q_{h} ; \tilde{q}\right):=D_{\tilde{q}}\left[\Pi\left(q_{h}\right)\right]=0, \quad \forall \tilde{q} \in \mathrm{CG}_{1} \times\left[\mathrm{CG}_{2}\right]^{2} \times \mathrm{NED}_{1} \times \mathrm{NED}_{1},
$$

which gives:

$$
F\left(q_{h} ; \tilde{q}\right)=a^{b}\left(w_{h}, \theta_{h} ; \tilde{w}, \tilde{\theta}\right)+a^{s}\left(\gamma_{h} ; \tilde{\gamma}\right)+a^{\Pi_{h}}\left(w_{h}, \theta_{h}, p_{h} ; \tilde{\gamma}\right)+a^{\Pi_{h}}\left(\gamma_{h} ; \tilde{w}, \tilde{\theta}, \tilde{p}\right)-W_{\mathrm{ext}}(\tilde{q})=0
$$

where:

$$
\begin{aligned}
a^{b}\left(w_{h}, \theta_{h} ; \tilde{w}, \tilde{\theta}\right) & =\int_{\omega} M\left(w_{h}, \theta_{h}\right) \cdot k(\tilde{w}, \tilde{\theta}) \mathrm{d} x, \\
a^{s}\left(\gamma_{h} ; \tilde{\gamma}\right) & =\int_{\omega} T\left(\gamma_{h}\right) \cdot \tilde{\gamma} \mathrm{d} x, \\
a^{\Pi_{h}}\left(w_{h}, \theta_{h}, \gamma_{h} ; \tilde{p}\right) & =\sum_{E \in \mathcal{E}_{h}} \int_{E}\left(\gamma\left(w_{h}, \theta_{h}\right) \cdot t-\gamma_{h} \cdot t\right) \cdot(\tilde{p} \cdot t) \mathrm{d} s .
\end{aligned}
$$


Deriving the Jacobian involves taking the Gâteaux derivative again, this time at a point $q_{h}$ in the direction of trial functions $q:=(w, \theta, \gamma, p)$ :

$$
J\left(q_{h} ; q ; \tilde{q}\right)=D_{q}\left[F\left(q_{h} ; \tilde{q}\right)\right] .
$$

Because the Reissner-Mindlin equations are linear, this operation is trivial, giving:

$$
J\left(q_{h} ; q ; \tilde{q}\right)=a^{b}(w, \theta ; \tilde{w}, \tilde{\theta})+a^{s}(\gamma ; \tilde{\gamma})+a^{\Pi_{h}}(w, \theta, p ; \tilde{\gamma})+a^{\Pi_{h}}(\gamma ; \tilde{w}, \tilde{\theta}, \tilde{p}) .
$$

Using a standard Newton method, given an initial guess $q^{0}$ we seek for $k=0, \ldots, n$ solutions $\delta q:=(\delta w, \delta \theta, \delta \gamma, \delta p)$ to the linear systems:

$$
\begin{array}{r}
J\left(q^{k} ; q ; \tilde{q}\right) \delta q=-F\left(q^{k} ; \tilde{q}\right), \\
q^{k+1}=q^{k}+\delta q .
\end{array}
$$

The left and right-hand side of the discrete Newton linear system corresponding to (29) and (27) respectively, has the following block structure:

$$
\left[\begin{array}{ccc}
A & 0 & C \\
0 & B & D \\
C^{T} & D & 0
\end{array}\right]\left[\begin{array}{l}
\delta z \\
\delta \gamma \\
\delta p
\end{array}\right]=\left[\begin{array}{l}
b_{z} \\
b_{\gamma} \\
b_{p}
\end{array}\right],
$$

where $\delta z:=(\delta w, \delta \theta)$ and $A, B$ correspond to the matrices associated with bilinear forms $a^{b}$ and $a^{s}$ in (29), respectively. The matrix $D$ is a diagonal-form edge mass matrix arising from the bilinear form:

$$
-\sum_{E \in \mathcal{E}_{h}} \int_{E}(\gamma \cdot t) \cdot(\tilde{p} \cdot t) \mathrm{d} s
$$

and $C$ arises from the bilinear form:

$$
\sum_{E \in \mathcal{E}_{h}} \int_{E}\left(\gamma\left(w_{h}, \theta_{h}\right) \cdot t\right) \cdot(\tilde{p} \cdot t) \mathrm{d} s
$$

The notation $b_{(\cdot)}$ corresponds to a splitting of the residual vector into its sub-blocks on the functions $z, \gamma$ and $p$.

Solving for $\delta \gamma$ with row two of (32):

$$
\delta p=D^{-1}\left(b_{\gamma}-B \delta \gamma\right)
$$

and for $\delta \gamma$ with row three of (32)

$$
\delta \gamma=D^{-1}\left(b_{p}-C^{T} \delta z\right),
$$

and then eliminating $\delta \gamma$ from (35) using: (36) gives

$$
\delta p=D^{-1}\left(b_{\gamma}-B D^{-1} b_{p}+B D^{-1} C^{T} \delta z\right),
$$

before finally eliminating $\delta p$ from the first row of (32) using (37) and rearranging to give:

$$
\begin{aligned}
A_{s} \delta z & =b_{s}, \\
A_{s} & =\left(A+C D^{-1} B D^{-1} C^{T}\right), \\
b_{s} & =b_{z}+C D^{-1} B D^{-1} b_{p}-C D^{-1} b_{\gamma} .
\end{aligned}
$$




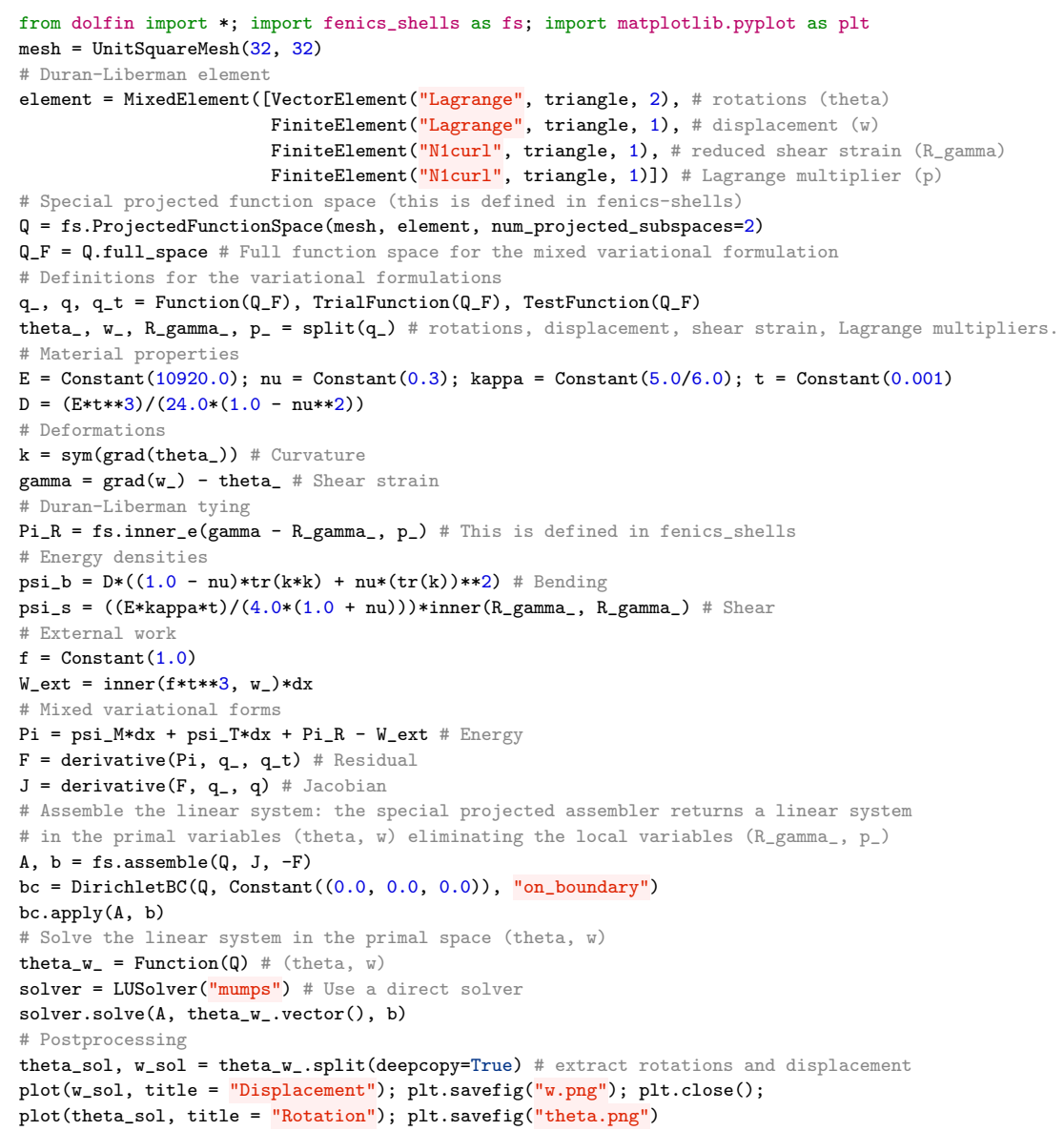

Figure 3: Complete Python code for solving clamped Reissner-Mindlin problem with uniform transverse loading.

Note again that $D$ is diagonal and can thus be cheaply inverted at the cell-local level allowing (38) to be formed locally as well. The above procedure is implemented at the element level using a custom assembly function written in $\mathrm{C}++$. FENICS automatically returns the dense cell-local version of the block structured system (32) corresponding to the Jacobian (29) and residual (27) forms. We then use the Eigen dense linear algebra package [35] to split up (32) into its constituent blocks before calculating $A_{s}$ and $b_{s}$ using (38). The cell-local contributions $A_{s}$ and $b_{s}$ are then assembled into a sparse matrix and vector associated with global displacement unknowns $\delta z$ only. Solving for the global unknown $\delta z$, we can then reconstruct the eliminated unknowns $\delta \gamma$ and $\delta p$ by post-processing at the cell-local level using (36) and (35), respectively.

A Python code showing how to define the Reissner-Mindlin problem in FENICS-SHELLS is given in Figure 3. The new ProjectedFunctionSpace class (line 9 in Figure 3) manages the relationship between full function space space with all unknowns $q_{h}:=\left(w_{h}, \theta_{h}, \gamma_{h}, p_{h}\right)$ the reduced function space unknowns with displacement unknowns $z=\left(w_{h}, \theta_{h}\right)$. The special custom assembly procedure in FENICS-SHELLS is invoked via a call to assemble passing the ProjectedFunctionSpace object as the first argument, resulting in the elimination of $\delta \gamma$ and $\delta p$ at the local level (line 34 in Figure 3).

The above procedure applies directly to the case of models with non-linear strain measures, e.g. 
the non-linear Naghdi shell model (see Section 2.1). The tying term in the Lagrangian is simply re-written using the appropriate strain measure $\gamma_{N}$ :

$$
\sum_{E \in \mathcal{E}_{h}} \int_{E}\left(\gamma_{N}\left(u_{h}, \beta_{h}\right) \cdot t-\gamma_{N h} \cdot t\right) \cdot\left(p_{h} \cdot t\right) \mathrm{d} s,
$$

before being consistently linearised at the symbolic level using the tools in UFL. The resulting discrete Newton system has precisely the same block form as (32), and so the same linear algebra assembly procedure can be used to eliminate the auxiliary unknowns $\gamma_{N_{h}}$ and $p_{h}$. We refer the reader to the documented demo Non-linear Naghdi roll-up cantilever [36] for an example applying MITC to a non-linear shell problem. Note, however, that we explicitly cure here only shear locking. Our high-level approach makes it relatively straightforward to directly apply MITC to user-specified thin structural models.

\subsection{Partial Selective Reduced Integration (PSRI)}

We use the PSRI approach to simultaneously deal with the shear- and membrane-locking issues in the shell-like models. Our method is inspired by, but not identical to the one shown in [7], which contains convergence proofs for the linear Naghdi model. The PSRI approach can be viewed as a reduced integration method[7], a stabilised mixed method [27], or an augmented Lagrangian method [21].

Following [7] we begin with the discrete linear Naghdi energy functional:

$$
\begin{aligned}
\Pi_{\tilde{N}}(u, \theta)=\frac{1}{2} \int_{\omega} N_{\tilde{N}}\left(u_{h}\right) \cdot e_{\tilde{N}}\left(u_{h}\right) \mathrm{d} x+\frac{1}{2} \int_{\omega} M_{\tilde{N}}\left(u_{h}, \theta_{h}\right) \cdot k_{\tilde{N}}\left(u_{h}, \theta_{h}\right) \mathrm{d} x & \\
& +\frac{1}{2} \int_{\omega} T_{\tilde{N}}\left(u_{h}, \theta_{h}\right) \cdot \gamma_{\tilde{N}}\left(u_{h}, \theta_{h}\right) \mathrm{d} x-W_{\text {ext }},
\end{aligned}
$$

or in shortened form:

$$
\Pi_{\tilde{N}}(u, \theta)=\Pi^{m}\left(u_{h}\right)+\Pi^{b}\left(u_{h}, \theta_{h}\right)+\Pi^{s}\left(u_{h}, \theta_{h}\right)-W_{\text {ext }},
$$

where $\Pi^{m}\left(u_{h}\right), \Pi^{b}\left(u_{h}, \theta_{h}\right)$ and $\Pi^{s}\left(u_{h}, \theta_{h}\right)$ correspond to the membrane, bending and shear energies of the linear Naghdi model, respectively.

The finite element spaces used in the construction of the PSRI element are second-order continuous piecewise-quadratic cubic-bubble-enriched Lagrangian elements for the displacements $u_{h} \in \mathcal{U}_{h}:=\left[C G^{2}+B_{3}\right]^{3}$ and second-order continuous piecewise-quadratic Lagrangian elements for the rotations $\theta \in \mathcal{R}_{h}:=\left[C G^{2}\right]^{2}$. The finite element space of cubic bubbles $B_{3}$ is defined by the Ciarlet triple $(T, \mathcal{V}, \mathcal{L})$ :

$$
T=\text { triangle, } \quad \mathcal{V}=\left\{v \in \mathcal{P}_{3}(T)|v|_{E}=0\right\}, \quad \mathcal{L}=v(1 / 3,1 / 3) .
$$

We then introduce a parameter $\alpha \in \mathbb{R}$ that splits the membrane and shear energy in the energy functional into a weighted sum of two parts:

$$
\Pi_{\tilde{N}}(u, \theta)=\Pi^{b}\left(u_{h}, \theta_{h}\right)+\alpha \Pi^{m}\left(u_{h}\right)+(1-\alpha) \Pi^{m}\left(u_{h}\right)+\alpha \Pi^{s}\left(u_{h}, \theta_{h}\right)+(1-\alpha) \Pi^{s}\left(u_{h}, \theta_{h}\right)-W_{\mathrm{ext}},
$$

to which we apply reduced integration to the parts weighted by the factor $1-\alpha$. PSRI is straightforward to implement in FEniCS using UFL [4]. We use a fourth-order accurate quadrature 
scheme on the portions of the energy in eq. 43 weighted by $\alpha$ and a second-order accurate quadrature scheme on the portion of the energy weighted by $1-\alpha$. As discussed in [7] a more refined interpretation of PSRI is a stabilised mixed formulation where the extra stress unknowns are projected out at assembly time. The lowest-order design in [7] suggests using a zero-order accurate (one-point) quadrature scheme, however in practice we have found this to produce spurious (zero-energy) modes.

The paper [7] also suggests setting $\alpha=1$. This choice is sufficient to obtain analytical convergence results. However, for the Reissner-Mindlin problem, this leads to suboptimal convergence rates [56], and a better choice in practice is setting $\alpha_{T}=\epsilon^{2} / h_{T}^{2}$ for each $T \in \mathcal{T}_{h}$. In numerical experiments we have found that the performance of the method is robust with respect to the parameter $\alpha$. The combination of a high-order polynomial space with a PSRI approach for alleviating membrane- and shear-locking seems both simple and effective.

\subsection{Curved Shells}

Finally, we describe how the curved shell geometry required for the linear and non-linear Naghdi models is implemented in FEniCS. We use an exact geometry approach, describing the mid-surface of the shell as a closed-form map $\phi_{0}: \omega \rightarrow \mathbb{R}^{3}$. For example, for a cylindrical geometry we have $\left(x_{0}, x_{1}\right) \in \omega:=[-\pi / 2, \pi / 2] \times[0, L]$ and the map $\phi_{0}=\left\{\rho \sin x_{0}, x_{1}, \rho \cos x_{0}\right\}$. From this, we can calculate approximations of the metric $a_{0}$ and curvature $b_{0}$ tensors and the normal $n_{0}$ of the middle surface from the map $\phi_{0}$ using UFL. Further derived quantities required, e.g. the contravariant components of the metric tensor $a_{0}^{\alpha \beta}$ and the elasticity tensor $A^{\sigma \beta \sigma \tau}$, can be straightforwardly expressed using UFL. An abbreviated Python code giving an indication for how the curved shell model can be implemented is given in Figure 4, extracted from the documented demo Clamped semi-cylindrical Naghdi shell under point load provided in the supplementary material.

\section{Examples}

To verify the convergence of the adopted discretisation techniques and illustrate the capabilities of the FENICS-SHELls library, we report some benchmark examples. We select eight test cases, including two linear Reissner-Mindlin plates (Section 4.1), two for linear Naghdi shells (Section 4.2), one for Marguerre-von Kármán shallow shells (Section 4.3), and three for nonlinear Naghdi shells (Section 4.4). The linear plate examples are discussed in more detail for testing the performance of our implementation of the MITC formulation; we also showcase here an adaptive remeshing example included in FENICS-SHELLS. For linear shells we consider both membrane- and bending-dominated regimes while the non-linear shell examples serve to check them in large displacements and rotation regimes and are based on the FENICS-SHELLS PSRI for curing the membrane and bending locking. The example on the thermal buckling of a Marguerre-von Kármán shallow shell of Section 4.3 is proposed here for the first time as a benchmark test of finite element shell models.

\subsection{Reissner-Mindlin plate}

In this section we demonstrate the correct convergence rate of our implementation of the Durán-Liberman element for the Reissner-Mindlin plate problem towards a manufactured analytical solution. Then, we show an implementation of a residual-based a posteriori error estimator which we use to drive mesh adaptivity, resolving the solution in a re-entrant plate problem with strong boundary layers. 


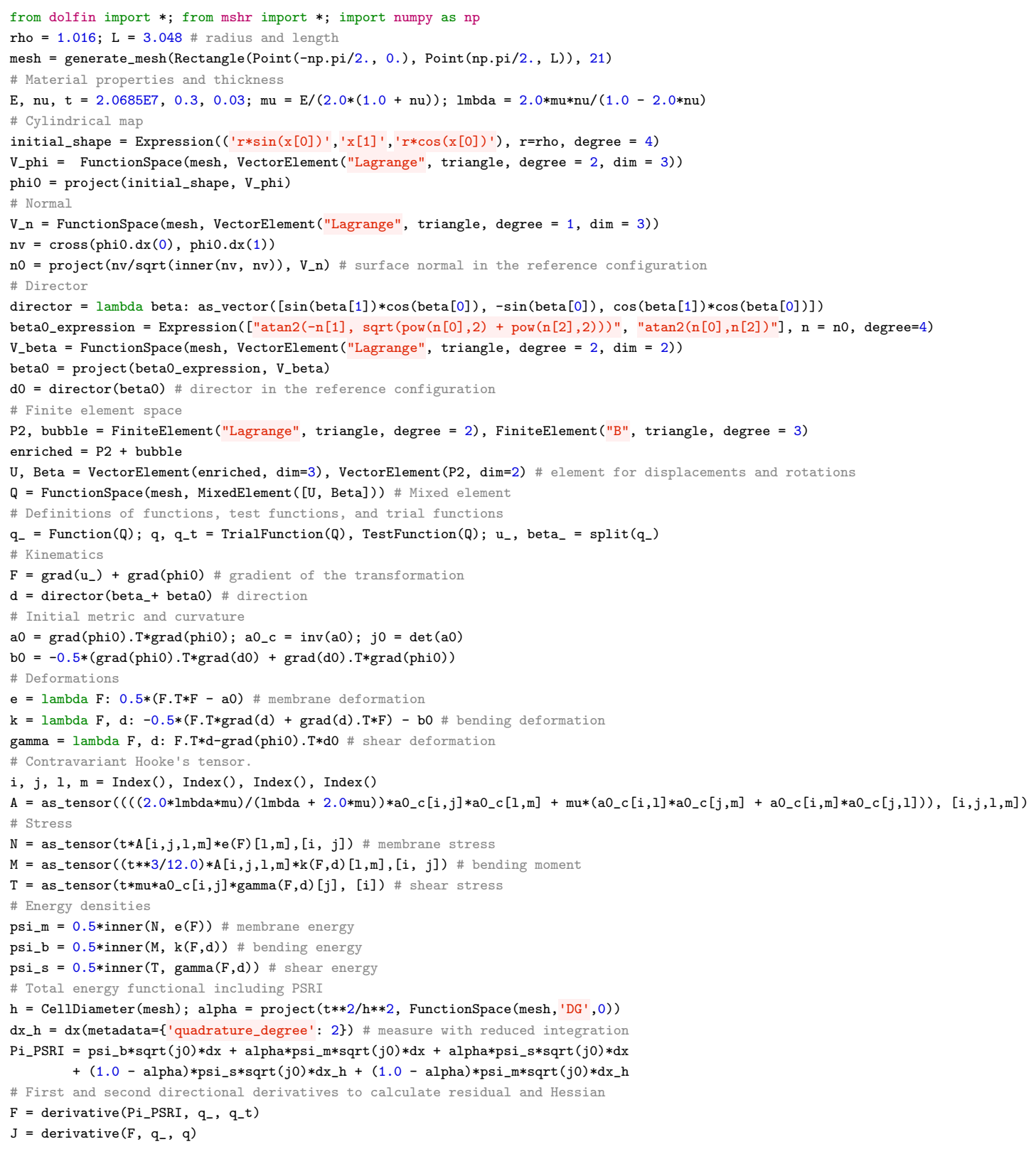

Figure 4: Abbreviated Python code showing construction of energies for half-cylinder non-linear Naghdi model (see Section 4.4). For further details we refer the reader to the documented demo Clamped semi-cylindrical shell under point load provided as the supplementary material. 

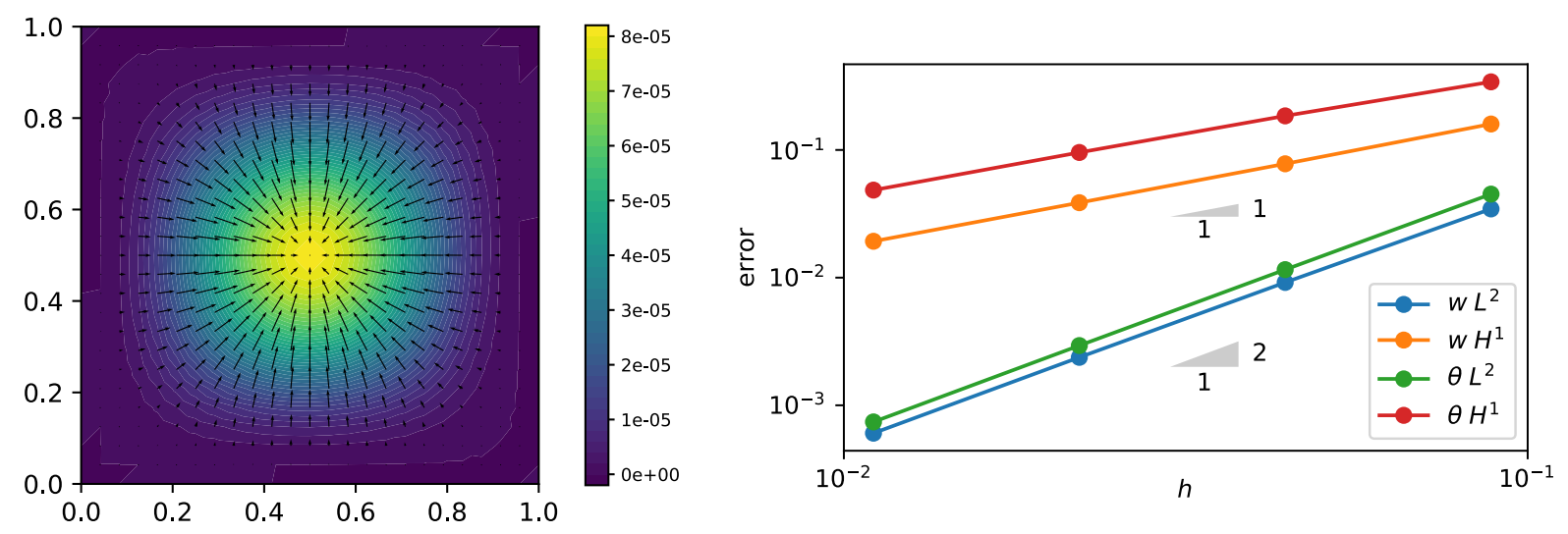

Figure 5: Chinosi clamped plate problem solved using Durán-Liberman element: Transverse displacements (scalar field, density plot) and rotations (vector field, quiver plot, $50 \%$ of points discarded) (left). Convergence to Chinosi analytical solution on sequence of uniformly refined meshes in $H^{1}$ and $L^{2}$ norms for $t=10^{-4}$ (right).

\subsubsection{Clamped square plate}

We solve the problem of a unit-square Reissner-Mindlin plate $\Omega=(0,1)^{2}$ clamped with uniform thickness $t=\epsilon=10^{-4}$, and with isotropic linear elastic material behaviour with Youngs modulus $E=10920$ and Poisson ratio $\nu=0.3$. The plate is clamped on the boundary (homogeneous Dirichlet boundary conditions on the transverse displacements and rotations) and loaded by a uniform pressure. The closed-form polynomial expressions for the loading and the resulting solution fields are given in Appendix A.

Figure 5 shows the solution to the above problem obtained with FENICS-SHELLS using a $32 \times 32$ cross-pattern mesh (left) and the error with respect to the analytical solution on a sequence of uniformly refined meshes in both the $H^{1}$ and $L^{2}$ norms (right). The convergence result given in the original paper of Durán and Liberman [32] is:

$$
\left\|\theta-\theta_{h}\right\|_{H^{1}\left(\omega, \mathbb{R}^{2}\right)}+\left\|w-w_{h}\right\|_{H^{1}(\omega)} \leq C h\|f\|_{L^{2}(\omega)},
$$

where $C$ is some unknown positive constant independent of the cell size $h$, corresponding to a linear convergence in the $H^{1}$-norm for both the transverse displacements and rotation variables. Although no proof is given in [32] we achieve quadratic convergence for both variables in the $L^{2}$ norm, the canonical 'one order higher convergence in $L^{2}$, behaviour. Figure 6 shows the convergence in the $H^{1}$-norm of the transverse displacement $w$ and the rotations $\theta$ for varying thickness $t$. We see a very slight deterioration of the error for a thick plate, but the rate of convergence is nearly identical for thick through to very thin plates.

In conclusion, this benchmark indicates that the implementation of the Durán-Liberman element in FENICS-SHELLS is locking-free and optimally convergent. We remark that a finite element method designed without consideration for locking would not converge at all for this problem. A commented code for this test case is given in the documented demo Clamped Reissner-Mindlin plate under uniform load (see supplementary material).

\subsubsection{Partly-clamped L-shaped plate}

This example shows how sophisticated and efficient adaptive remeshing strategies can be easily implemented using FEnICS-SHELLS. We consider the a posteriori error estimator proposed in [16], 

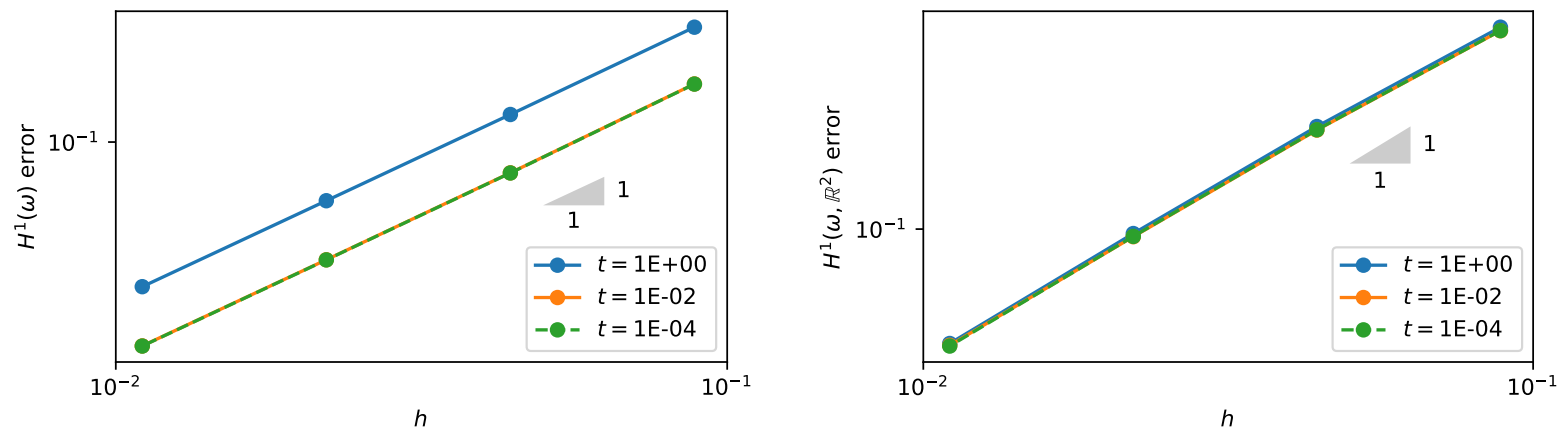

Figure 6: Convergence of Reissner-Mindlin problem discretised with Durán-Liberman element to Chinosi analytical solution for varying thickness parameter $t$. Left: Error for the transverse displacement $w\left(H^{1}\right.$-norm) as a function of the mesh size $h$. Right: Error for the rotation field $\theta$ ( $H^{1}$-norm). In the latter case all lines lie on top of each other (convergence behaviour independent of $t$ ). We achieve optimal convergence in all cases.

which is similar to the one developed in [24], but it includes extra terms that control the error at boundaries induced by the strong boundary layer present [8] in the Reissner-Mindlin problem as $\epsilon \rightarrow 0$. The full specification of the error indicator is given in Appendix B. It is possible to implement these complex expressions in UFL with just a few lines of code and a demo in FEnICS-ShELLS demonstrates the procedure.

We consider the problem of a non-convex L-shaped plate with a re-entrant corner clamped along the two edges that form the re-entrant corner, and the other four sides left free, Figure 7. The plate is loaded with a uniform transverse load $f$ scaled with the cube of the plate thickness $t^{3}$. The material parameters are the same as before. The difficulties of resolving the low regularity solution in the straight corners and in the re-entrant corner, in addition to the strong boundary layers on the free edges, means that a uniform refinement strategy will be very inefficient. Using an a posteriori error estimator to guide the refinement process can lead to orders of magnitude efficiency improvements.

Because no exact analytical solution is known for the studied problem we cannot calculate the exact error. Therefore we resort to comparing the behaviour of the error estimator under both uniform and adaptive refinement strategies.

Figure 8a shows the convergence of the error estimator for a moderately thick plate $t=10^{-1}$ using adaptive (blue solid) and uniform (green dashed) refinement. We can see that using uniform refinement we achieve an algebraic convergence rate of only $\mathcal{O}\left(d^{-1 / 4}\right)$ where $d$ is the number of degrees of freedom for the discrete system. This corresponds to a classical convergence rate of $\mathcal{O}\left(h^{1 / 2}\right)$. This sub-optimal behaviour is expected given the low regularity of the solution particularly in the re-entrant corner. The convergence of the adaptive scheme initially matches that for the uniform refinement before increasing to $\mathcal{O}\left(d^{-1 / 2}\right)$ due to the strong local refinements in the re-entrant corner region, and also in the other corners during the latter refinement steps. In Figure 7a we show the meshes at the 2 nd and 6th adaptive refinements, with 2,499 and 10,494 degrees of freedom, respectively. The strong refinement near the re-entrant corner is clearly evident.

Critically, we can achieve the same level of convergence with significantly fewer degrees of freedom using the adaptive scheme. Taking the most refined uniformly meshed problem, with $2,365,443$ degrees of freedom, we can reach the roughly the same level of error using 123,495 degrees of freedom with the adaptive strategy. 


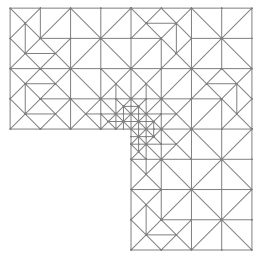

(a) Thick plate $\left(t=10^{-1}\right)$
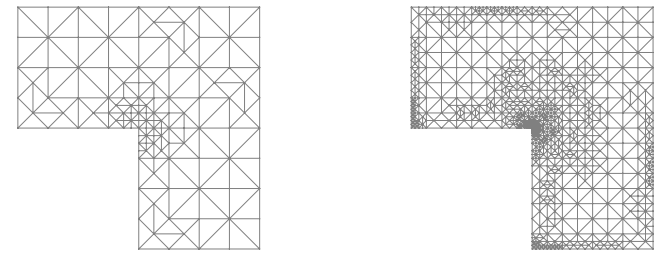

(b) Thin plate $\left(t=10^{-4}\right)$

Figure 7: Meshes after adaptive remeshing at different refinement levels (3rd and 6th level) for thick and thin plates. The increased refinement on the free boundaries for thin plates capture the strong boundary layer effect as $t \rightarrow 0$.

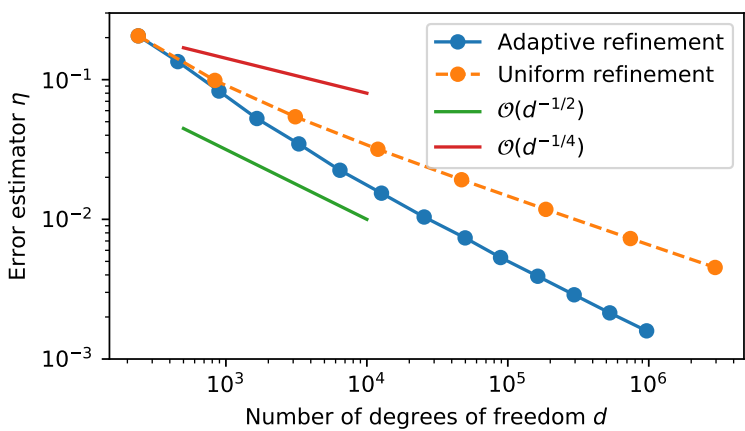

(a) Thick plate $\left(t=10^{-1}\right)$

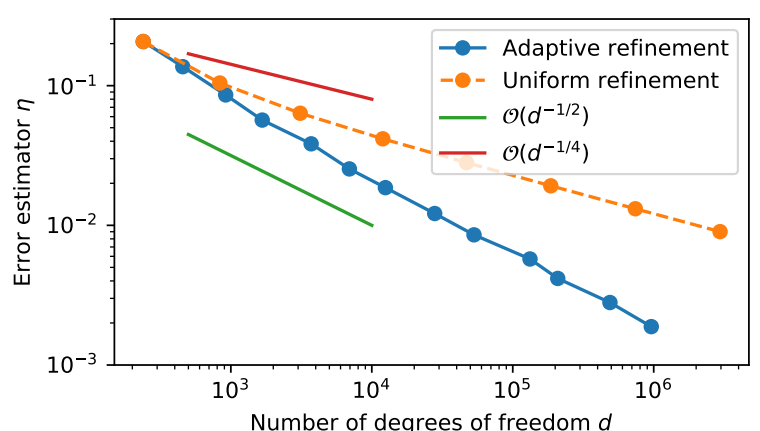

(b) Thin plate $\left(t=10^{-4}\right)$

Figure 8: Plot of error estimator $\eta$ against the number of cells in the L-shaped plate problem with using adaptive and uniform refinement. The rate of convergence is significantly improved using the adaptive refinement strategy compared with a uniform refinement strategy.

The trends for a very thin plate with $t=10^{-4}$ shown in Figure $8 \mathrm{~b}$ and Figure $7 \mathrm{~b}$ are broadly similar to those in Figure 7a. However, in addition, because of the presence of the strong boundary layer on the free edges as $t \rightarrow 0$, we can see that the error indicator preferentially refines these regions as well as the re-entrant corner.

\subsection{Linear Naghdi shell}

In this section we demonstrate the performance of the finite element discretisation of the Naghdi linear shell model implemented in the FENICS-SHELls library. The numerical results presented here are based the PSRI formulation. To verify the discretisation in both membrane- and bending dominated regimes two well known benchmark examples have been chosen, namely, a partly clamped hyperbolic paraboloid (bending-dominated test) and an axisymmetric hyperboloid with clamped ends (membrane-dominated test). In both cases, the computational domain is a rectangular domain $\omega$ which is mapped to the surface in $\mathbb{R}^{3}$ through the action of the map $\phi_{0}$.

\subsubsection{Partly clamped hyperbolic paraboloid}

We consider a thin shell whose middle surface is an hyperbolic paraboloid; the shell is clamped on one of its edges and subject to a uniform load in the vertical direction, see Figure 9 for details. This is an excellent bending dominated benchmark problem to test a FE formulation with respect 

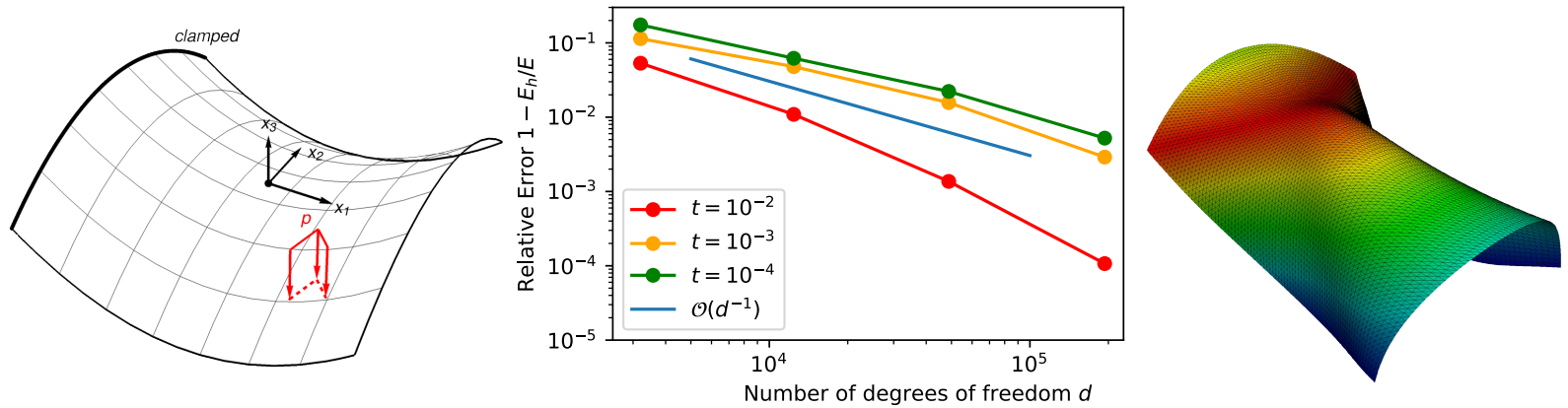

Figure 9: Partly clamped hyperbolic paraboloid with linear Naghdi shell model: initial shape (left), convergence plot (centre), and deformed shape (right). We obtain convergence of $\mathcal{O}\left(d^{-1}\right)$ in the energy for this problem.

to membrane-locking issues [15]. The initial shape is the analytical surface $\phi_{0}=\left\{x_{0}, x_{1}, x_{0}^{2}-x_{1}^{2}\right\}$ with $\left(x_{0}, x_{1}\right) \in[-1 / 2,1 / 2] \times[-1 / 2,1 / 2]$. For the simulation we set Poisson ratio to be $\nu=0.3$. The convergence analysis is performed for thicknesses $t=10^{-n}$ for $n=1,2,3,4$ and obtained by setting the uniform pressure load to $p$ to be proportional to $t$. Convergence rates in strain energy are reported for different values of the dimensionless thinness parameter $t=\epsilon$. The deformed shape of the shell shows two zones corresponding to radically different shell behaviours: a zone where geometry and constraints prevent pure bending displacements (inhibited zone) and a zone where they are allowed (non-inhibited zone). For very thin shells this implies a sharp change of the shell stiffness across the boundary separating the two zones, born out by the bending energy localisation [52].

\subsubsection{Axisymmetric hyperboloid with clamped ends}

We consider a thin shell whose middle surface is an hyperboloid of revolution; the shell is clamped on both its edges and subjected to a surface axisymmetric normal pressure, so that symmetry arguments enable us to solve the problem over a smaller computational domain (1/8th of the whole domain), see Figure 10 for details. This is a well-posed membrane dominated benchmark problem [41]. The initial shape is the analytical surface $\phi_{0}=\left\{\cos x_{0} \cosh x_{1}, \sin x_{0} \cosh x_{1}, \sinh x_{1}\right\}$ with $\left(x_{0}, x_{1}\right) \in[0, \pi / 2] \times[-1,0]$. For the simulation we set Poisson ratio $\nu=1 / 3$ and the pressure load $f=f_{0} \cos \left(2 x_{0}\right)$. Convergence rates in strain energy are reported for different values of the thinness parameter $t$. The deformed shape of the shell shows a boundary layer of width $\approx 6 \sqrt{t}$ near the clamped edge can be recognised, where the mesh must adequately refined for proper convergence. These results are in agreement to what is reported in the literature [41].

\subsection{Marguerre-von Kármán shallow shell}

We discuss the performance finite element discretisations of the nonlinear Marguerre-von Kármán shallow shell model (see Section 2.2) implemented in the FENICS-SHELLS library. We compare here the results obtained with the MITC and PSRI formulations.

\subsubsection{Lenticular plate with inelastic curvature}

We consider an initially flat isotropic linear elastic disk of radius $a$ with lenticular thickness subject to a through-the-thickness temperature gradient. Thus, the initial shape of the plate is the 

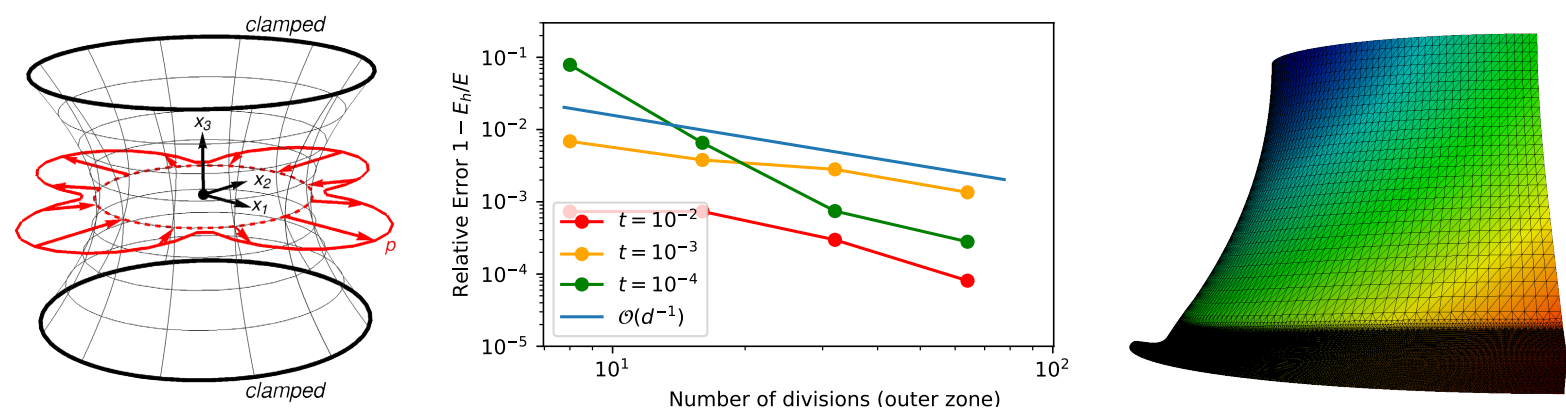

Figure 10: Clamped hyperboloid: initial shape (left), convergence plot (centre), and deformed shape (right). We obtain convergence of $\mathcal{O}\left(d^{-1}\right)$ in the energy for this problem. This convergence requires strong refinement in the region of the boundary layer, shown on the deformed shape (right).

disk $x_{0}^{2}+x_{1}^{2} \leq a^{2}$ and the thickness varies as $\left(1-x_{0}^{2} / a^{2}-x_{1}^{2} / a^{2}\right) t$, where $t$ is the maximal thickness at the center. This special shape has been proposed by Mansfield[59, 60] to avoid boundary layer effects and obtain a prestressed plate allowing for an exact analytical solution in the nonlinear regime. The temperature gradient is modelled as a uniform isotropic inelastic curvature $\bar{k}$ with $\bar{k}_{x x}=\bar{k}_{y y}=k_{T}$ and $\bar{k}_{x y}=0$ (with $x_{0}=x, x_{1}=y$ ). Mansfield has shown that in the framework of the von Kármán model the exact solution of the problem is with uniform curvature in space. The curvature tensor at the equilibrium is with $k_{x y}=0$ and

$$
\left\{\begin{array}{l}
\text { for } k_{T} \leq k_{T}^{*}, \quad k_{x}=k_{y}: \quad k_{x}+\frac{4}{(1+\nu)^{2}} \frac{k_{x}^{3}}{k_{T}^{* 2}}=k_{T} \\
\text { for } k_{T}>k_{T}^{*}, \quad k_{x}, k_{y}=k_{T} \pm \sqrt{k_{T}^{2}-k_{T}^{* 2}}
\end{array} \quad, \quad k_{T}^{*}=\frac{t}{r^{2}} \frac{2}{1+\nu} \sqrt{\frac{2(7+\nu)}{(1+\nu)}} .\right.
$$

For $k_{T} \ll k_{T}^{*}$, the behaviour is approximately linear. The equilibrium curvature $k$ is spherical and close to $\bar{k}$. However, conforming the plate to a spherical surface implies membrane extension. For $k_{T} \gg k_{T}^{*}$, the extensional deformation becomes too expensive from the energetic point of view and the minimal energy configurations are approximately cylindrical and bending-dominated. The critical value $k_{T}^{*}$ corresponds to a bifurcation from the spherical shape. For $k \simeq k_{T}^{*}$ bending and extensional energies are of the same order. In the perfectly isotropic case, for $k>k_{T}^{*}$ the plate is neutrally stable: all the shapes obtained by rotating the curvature axis are still solutions of the problem. This structure was a source of inspiration for several recent works on morphing plates and shells $[84,85,40]$.

We perform simulations increasing the inelastic curvature $k_{T}$, for a Poisson ration $\nu=0.3$ and maximum thickness $t=0.01$. The Young modulus has no influence here. To break the rotational symmetry, we introduce a slight imperfection in the inelastic curvature imposing $\bar{k}_{x}=k_{T} / \rho$, $\bar{k}_{y}=k_{T} \rho$ with $\rho=0.998$. Figure 11 shows the bifurcation diagrams obtained with the MITC and the PSRI discretisations for the shearable version of the model exposed in Section 2.2. We use uniform unstructured triangular meshes and parametrise the mesh size by the number of division $n$ along the radius. To avoid rigid body motions, we applied suitable pointwise Dirichlet boundary conditions. Namely we set to zero all the components of the displacement at the center, the displacement in $x$ direction at $(0, a)$ and the displacement in $y$ direction at $(a, 0)$. The analytical solution (45) is reported in the plot as solid blue line. The results obtained with both the discretisations converge to the analytical solution. However, PSRI converges more quickly than 
MITC, giving very good results even with $n=5$. A possible explanation is that membrane locking is not cured in our MITC implementation. We conclude that the PSRI technique introduced in FENICS-SHELLS is a very efficient discretisation to cure both shear and membrane locking in the weakly nonlinear regime. This will be further tested and assessed in the next Section. To introduce fully nonlinear models, we compare in Figure 12 the results obtained with the fully nonlinear Naghdi shell model and the Marguerre-von Kármán shallow shell model. In both cases we use the PSRI discretisation. As expected [53], the results confirm that Marguerre-von Kármán model can safely be used to approximate the Naghdi model for sufficiently small loadings, namely for curvatures of the order of $a^{2} / t$. More surprisingly, for the present test the discrepancies with respect the fully nonlinear model remain tolerable also for $k \simeq 50 a^{2} / t$. The documented demo Buckling of a heated von Kármán plate in the supplementary material includes the full commented code for the Marguerre-von Kármán shallow shell with PRSI discretisation. An extension of this problem to the case of an orthotropic material is included in FENICS-SHELLS as a further documented demo in order to illustrate some basic tools for modelling composite laminates that we do not explicitly describe in this paper.
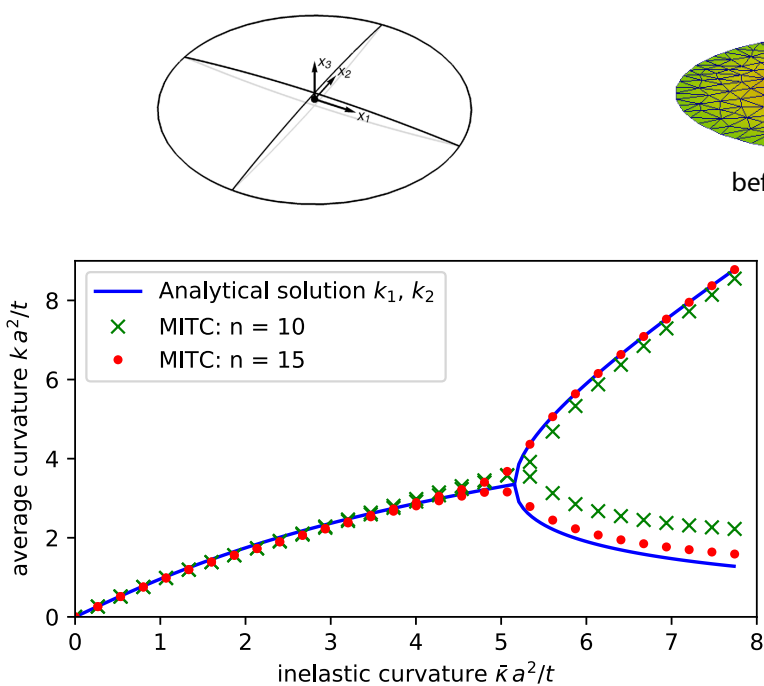
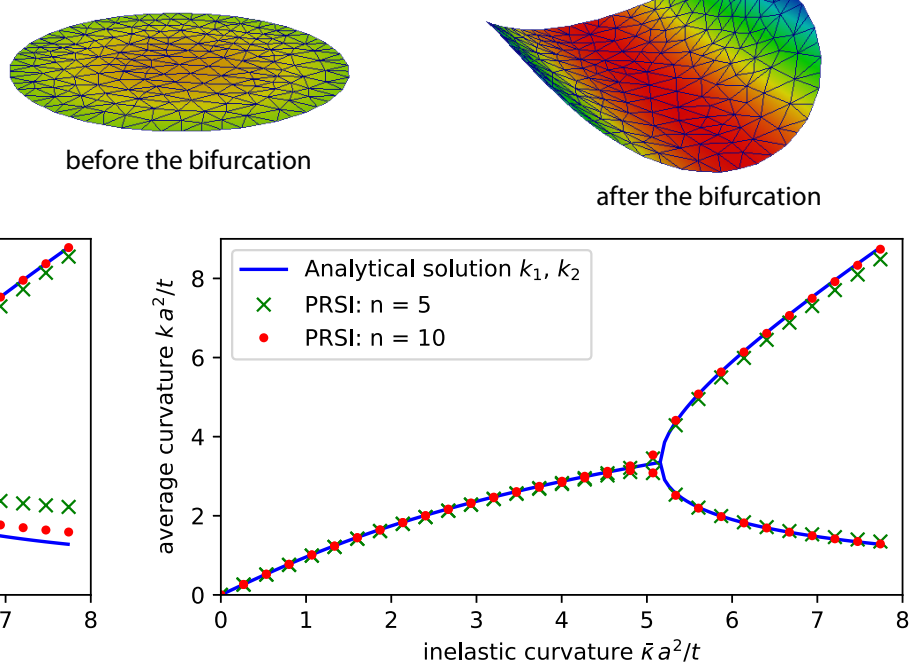

Figure 11: Lenticular orthotropic plate. Bottom row: Bifurcation diagram for the curvatures $k_{x}$ and $k_{y}$ at the equilibrium as a function of the temperature loading. The curvature values reported in the plots as dots are the spatial average extracted from the finite element results with the MITC (left) and PRSI (right) models with uniform unstructured meshes with $n \in[5,10]$ divisions along the radius. The top row: Sketch of the lenticular plate (left) and snapshots of the equilibrium shapes before (center) and after (right) the bifurcation. The results are for $a^{2} / t=100$ and $\nu=0.3$

\subsection{Nonlinear Naghdi shell}

We demonstrate here the performance of the finite element discretisation of the nonlinear Naghdi shell model implemented in the FENICS-SHELLS library. To test the predictive capability of the formulation in the large displacements and rotations regime we selected three well known benchmark problems, see [43] and references therein. 


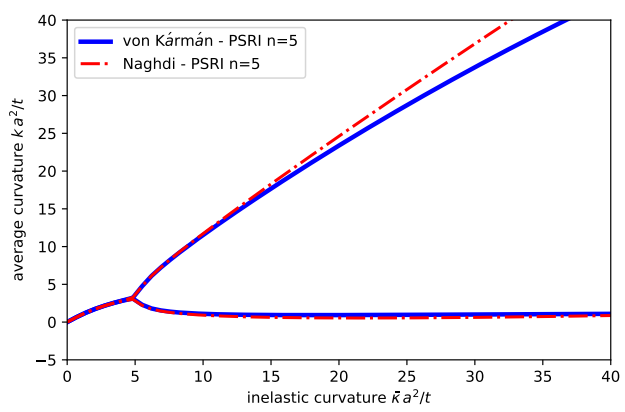

Figure 12: Lenticular orthotropic plate. Comparison of the results obtained with the von Kármán and the Naghdi models using the PSRI discretisation and an unstructured uniform mesh with $n=5$ division along the radius. The results are for $a^{2} / t=100$ and $\nu=0.3$.

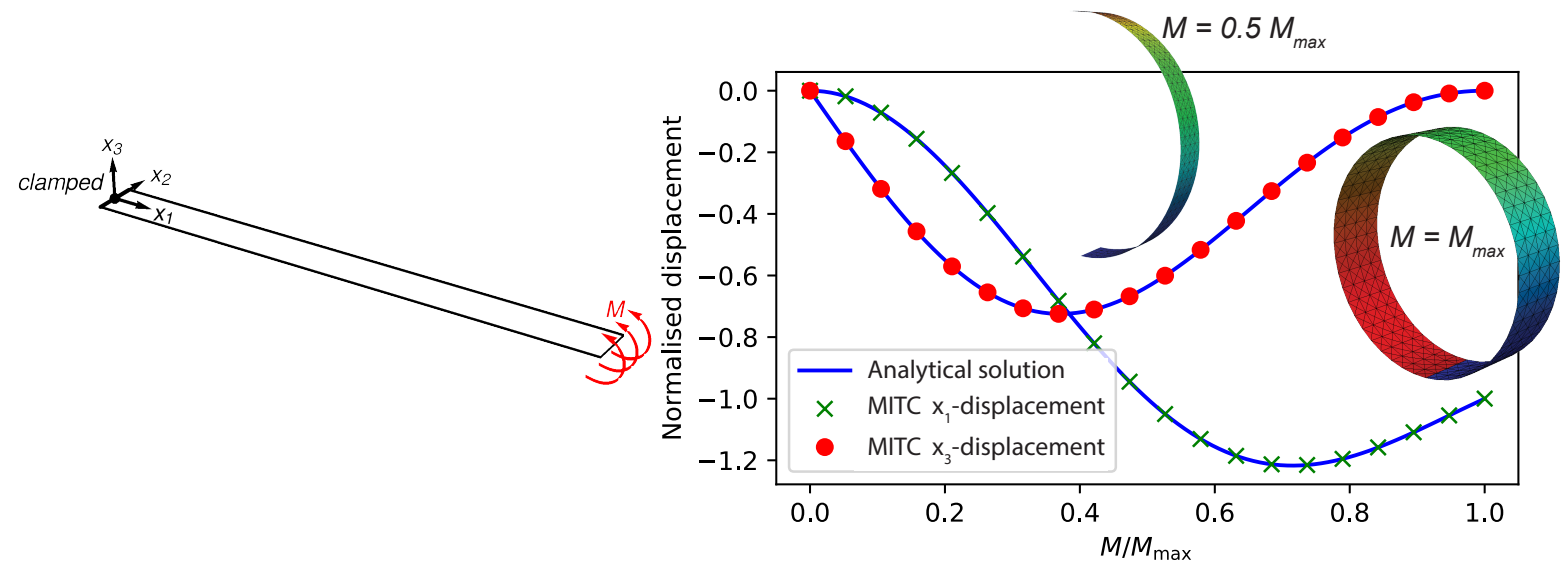

Figure 13: Cantilever plate under end moment. Left: initial geometry and loading. Right: Comparison between analytical and numerical solution (MITC) for the moment-displacement diagram, where the insets show the deformed configurations for $M=0.5 M_{\max }$ and $M=M_{\max }$. We report here the results for displacement at the free end in the $X_{1}$ and $X_{3}$ directions, normalised with the initial length $L$.

\subsubsection{Cantilever plate under end moment}

We consider a strip clamped on one of its edges and subjected to a moment $M$ on the other edge, see Figure 13 for details. In its natural configuration the shell occupies a flat rectangular domain of width $b$, length $L$. In the numerical simulations we set $L=12 b$, thickness $t=b / 10$, and Poisson ratio $\nu=0$. For load $M=2 \pi E I / L$, where $I=t^{3} / 12$ the plate completely rolls up on itself. The normalised vertical and horizontal displacements at the free end have the analytical expressions $w / L=(1-\cos 2 \pi \mu) /(2 \pi \mu)$ and $v / L=\sin (2 \pi \mu) /(2 \pi \mu)-1$, respectively, where $\mu=M / M_{\max }$. A comparison between the finite element and analytical solutions in terms of load-deflection curves is reported in Figure 13. The numerical solution is computed by discretising the domain with a $48 \times 4$ element mesh and shows excellent agreement with the analytical one. The insets show the deformed configurations of the plate for two values of the end moment. 


\subsubsection{Slit annular plate under end shear force}

We consider a slit annular plate of internal radius $R_{1}=6$ and external radius $R_{2}=10$ clamped on one of its edges and subjected to a line shear load $p$ on the other edge, see Figure 14 for details. A comparison between the finite element and reference solutions in terms of load-deflection curves is
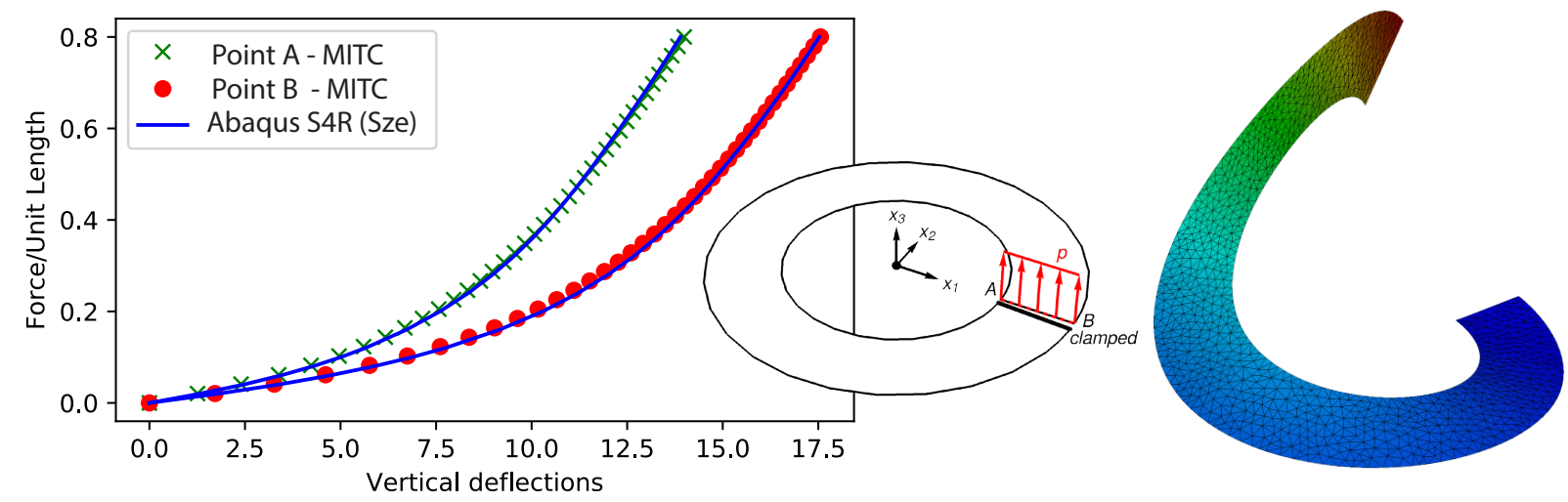

Figure 14: Slit annular plate subjected to a line vertical load. The load-deflection diagram compare the numerical solution obtained with FEnICS-ShELLS MITC discretisation with a reference solution (Sze [87], Abaqus S4R). Right: geometry and loading; Left: final deformed configuration. Excellent agreement between the result of Sze [87] and FENICS-SHELLS is achieved.

reported in Figure 14, where we set the thickness $t=0.03$, Poisson ratio $\nu=0$ and maximum load $p=0.8 E$, with $E=21 \times 10^{6}$. The numerical solution is computed by discretising the domain with a 28-division unstructured mesh and the MITC technique. The reference solution [87] is computed on a $10 \times 80$ AbAQUS S4R-element mesh. We see excellent agreement between the two results.

\subsubsection{Clamped semi-cylindrical shell under point load}
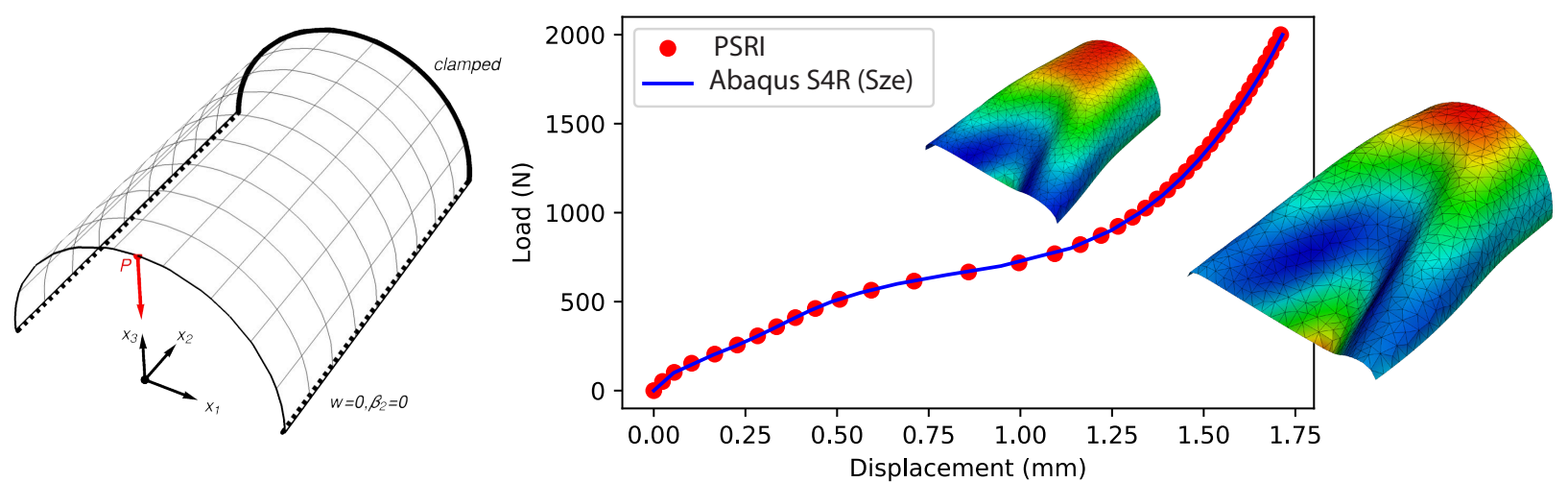

Figure 15: Clamped semi-cylindrical shell under point load. Left: Initial configuration and boundary conditions. Right: Comparison of the results of the PRSI implementation with a reference solution (Sze [87], Abaqus S4R). The insets show the deformed configurations for $P=P_{\max }$ and $P=P_{\max } / 2$. Notice the stiffening of the shell beyond a load of around $750 \mathrm{~N}$. 
We consider a semi-cylindrical shell clamped on one of its curved edges and subjected to a point load $P$ at the centre of the free curved edge. Along its straight longitudinal edges the transverse displacement and the normal rotation are restrained, see Figure 15 for details.

The computational domain is a rectangle $\left(x_{0}, x_{1}\right) \in[-\pi / 2, \pi / 2] \times[0,3.048]$ which is mapped to the natural configuration semi-cylindrical shell of radius $\rho=1.016$ through the initial transformation $\phi_{0}\left(x_{0}, x_{1}\right)=\left\{\rho \sin x_{0}, x_{1}, \rho \cos x_{0}\right\}$. A comparison between the $\mathrm{FE}$ and reference solution in terms of load-deflection curves is reported in Figure 15 for the thickness $t=0.03$, Young modulus $E=2.0685 \times 10^{7}$, Poisson ratio $\nu=0.3$, and a maximum load $P_{\max }=2000$. The numerical solution is computed by discretising the domain with a 21 -division unstructured mesh and shows a very good agreement with the reference solution in [87] computed on a $40 \times 40$ ABAQUS S4R-element mesh. For a complete commented code for this test case we refer to the documented demo Clamped semi-cylindrical Naghdi shell under point load, available in the supplementary material.

\section{Conclusions}

In this paper we shown how a high-level approach to specifying thin structural models, in conjunction with automatic code generation tools, can lead to simple, extensible and effective finite element plate and shell formulations. The power of this approach is demonstrated via the open-source FENICS-SHELLS library. We have shown the main mathematical aspects of the structural models available and the numerical procedures used to discretise them, with a particular emphasis on shear- and membrane-locking issues. We selected two main discretisation strategies: a special implementation of the MITC technique that provides an effective cure for shear-locking in linear plate models, and an extension to nonlinear shells of the PRSI strategy proposed in [7] for nonlinear shell problems shear- and membrane-locking. The examples section, accompanied by a set of documented demos, while by no means exhaustive, shows the good performance of the proposed methods and the type of analysis that can be performed.

We believe that FENICS-SHELLS will be a helpful contribution for researchers and graduate students in the community simulating thin structures. FENICS-SHELLS could provide users with efficient and simple tools to implement specific solvers for multilayered or other specific plate and shell models [80], shell models including multiphysical couplings or nonlinear material behaviour [79, 48, 40], as well as one atom thick shell structures such as nanotubes and graphene [9].

The present work constitutes an instrumental step in a larger project on the mechanics and physics of shell structures, including the analysis of the nonlinear material behaviour and fracture of isotropic and anisotropic shells through phase-field variational models [22, 42, 63, 6], applications to multistable structures with embedded active materials [40, 83], and for the understanding of singularities and energy scalings in plates and shells [30]. Current and future technical developments of FENICS-SHELLS will be aimed at providing anisotropic adaptive remeshing tools [82,64] and coupling to advanced nonlinear solvers, such as asymptotic numerical continuation methods [88] and deflation techniques [33] to automatically detect multiple solutions of nonlinear systems. A further interesting extension would be to adapt to shell modelling the special approach proposed by [78] for solving PDEs on manifolds with FEnICS. 


\section{Appendix A. Closed-form solution for a Reissner-Mindlin clamped square plate with polynomial loading}

This analytical solution is taken from the paper [27]. With transverse loading:

$$
\begin{aligned}
f(x, y) / D=12 y(y-1) & \left(5 x^{2}-5 x+1\right)\left\{2 y^{2}(y-1)^{2}+x(x-1)\left(5 y^{2}-5 y+1\right)\right\} \\
+ & 12 x(x-1)\left(5 y^{2}-y+1\right)\left\{2 x^{2}(x-1)^{2}+y(y-1)\left(5 x^{2}-5 x+1\right)\right\},
\end{aligned}
$$

with $D=E t^{3} /\left[12\left(1-\nu^{2}\right)\right]$, it is possible to obtain the following closed-form solutions for the rotations and transverse displacements:

$$
\begin{aligned}
& \theta(x, y)=\left.\left\{y^{3}(y-1)^{3} x^{2}(x-1)^{2}(2 x-1), x^{3}-1\right) y^{2}(y-1)^{2}(2 y-1)\right\}^{T} \\
& w(x, y)= \frac{1}{3} x^{2}(x-1)^{3}(y-1)^{3}-\frac{2 t^{2}}{5(1-\nu)}\left[y^{3}(y-1)^{3} x(x-1)\left(5 x^{2}-5 x+1\right)\right. \\
&\left.\quad+x^{3}(x-1)^{3} y(y-1)\left(5 y^{2}-5 y+1\right)\right] .
\end{aligned}
$$

\section{Appendix B. Residual-based a posteriori error estimator}

The key result found in the paper[16] is that the error between the finite element solution and the exact solution defined in the following sense:

$$
\left\|| | ( \theta - \theta _ { h } , w - w _ { h } ) \left|\left\|_{h}+t|| \gamma-\gamma_{h}\right\|_{L^{2}(\Omega)}+\left\|\gamma-\gamma_{h}\right\|_{H^{-1}(\Omega)}+t^{2}\left\|\operatorname{rot}\left(\gamma-\gamma_{h}\right)\right\|_{L^{2}(\Omega)},\right.\right.
$$

where the mesh-dependant norm $\|\mid(\theta, w)\| \|_{h}$ is defined by:

$$
\|\mid(\theta, w)\|\left\|_{h}^{2}=\right\| \theta\left\|_{H^{1}(\Omega)}^{2}+\sum_{T \in \mathcal{T}_{h}} \frac{1}{t^{2}+h_{T}^{2}}\right\| \nabla w-\theta \|_{L^{2}(T)}^{2},
$$

can be controlled by an error estimator of the following form:

$$
\begin{aligned}
\eta_{h}^{2} & =\sum_{T \in \mathcal{T}_{h}}\left(\eta^{2}+\rho^{2}\right)+\sum_{E \in \mathcal{E}_{h}^{\text {int }}} \eta_{\text {int }}^{2}+\sum_{E \in \mathcal{E}_{h}^{\text {free }}} \eta_{\text {free }}^{2}, \\
\eta^{2} & =h_{T}^{2}\left(h_{T}^{2}+t^{2}\right)\left\|f+\operatorname{div} \gamma_{h}\right\|_{L^{2}(T)}^{2}+h_{T}^{2}\left\|\operatorname{div} M_{h}+\gamma_{h}\right\|_{L^{2}(T)}^{2}, \\
\eta_{\text {int }}^{2} & =h_{E}\left(h_{E}^{2}+t^{2}\right)\left\|\llbracket \gamma_{h} \cdot n \rrbracket\right\|\left\|_{L^{2}(E)}+h_{E}\right\| \llbracket M_{h} n \rrbracket \|_{L^{2}(E)}^{2}, \\
\eta_{\text {free }}^{2} & =h_{E}\left\|M_{h} n\right\|_{L^{2}(E)}^{2}+h_{E}\left(h_{E}^{2}+t^{2}\right)\left\|\gamma_{h} \cdot n\right\|_{L^{2}(E)}^{2}, \\
\rho^{2} & =\left\|\operatorname{rot}\left(I-R_{h}\right) \theta_{h}\right\|_{L^{2}(T)}^{2}+\left\|\left(I-R_{h}\right) \theta_{h}\right\|_{L^{2}(T)}^{2},
\end{aligned}
$$

where $\mathcal{E}_{h}^{\text {free }} \not \subset \mathcal{E}_{h}^{\text {int }}$ are the subset of edges on the free boundary (natural boundary conditions).

\section{Acknowledgements}

We would like to thank Douglas N. Arnold for sharing his implementation of the unreduced Durán-Liberman element in the Unified Form Language with us.

Jack S. Hale is supported by the National Research Fund, Luxembourg, and cofunded under the Marie Curie Actions of the European Commission (FP7-COFUND) Grant No. 6693582. 
Stéphane P. A. Bordas thanks partial funding for his time provided by the European Research Council Starting Independent Research Grant (ERC StG) Grant No. 279578 entitled "Towards real time multiscale simulation of cutting in non-linear materials with applications to surgical simulation and computer guided surgery".

Matteo Brunetti and Corrado Maurini acknowledge the financial support of Project ANR-13JS09-0009 (Agence Nationale de la Recherche, 2014).

\section{Supporting information}

Code (LGPLv3), documentation and demos are available from the links at: https://doi.org/ 10.6084/m9.figshare.4291160.

Three documented demos are formally enclosed as supplementary materials:

(S1) Clamped-Reissner-Mindlin-plate-under-uniform-load.pdf

(S2) Buckling-heated-von-Karman-plate.pdf

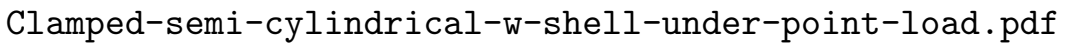

\section{References}

[1] Mat-fem: Learning the finite element method with matlab and gid, 2012. URL http://www.cimne.com/mat-fem.

[2] ABAQUS Theory Guide, Version 201\%. Simulia, 2016.

[3] M. Ainsworth and K. Pinchedez. The hp-MITC finite element method for the Reissner-Mindlin plate problem. Journal of Computational and Applied Mathematics, 148(2):429-462, November 2002. ISSN 0377-0427. doi: 10.1016/S0377-0427(02)00560-5. URL http://www. sciencedirect.com/science/article/B6TYH-472HT8V-9/ 2/006aa5725265e03487fd00f261e18249.

[4] M. Alnæs, J. Blechta, J. Hake, A. Johansson, B. Kehlet, Anders Logg, Chris Richardson, Johannes Ring, Marie E. Rognes, and Garth N. Wells. The FEniCS Project Version 1.5. Archive of Numerical Software, 3(100), December 2015. ISSN 2197-8263. doi: 10.11588/ans.2015.100.20553. URL http://journals.ub.uni-heidelberg.de/ index.php/ans/article/view/20553.

[5] M. S. Alnæs, A. Logg, K. B. Ølgaard, M. E. Rognes, and G. N. Wells. Unified Form Language: A Domain-specific Language for Weak Formulations of Partial Differential Equations. ACM Trans. Math. Softw., 40(2):9:1-9:37, March 2014. ISSN 0098-3500. doi: 10.1145/2566630. URL http://doi .acm.org/10.1145/2566630.

[6] F. Amiri, D. Millán, Y. Shen, T. Rabczuk, and M. Arroyo. Phase-field modeling of fracture in linear thin shells. Theoretical and Applied Fracture Mechanics, 69:102-109, February 2014. ISSN 0167-8442. doi: 10.1016/j.tafmec. 2013.12.002. URL http://www.sciencedirect.com/science/article/pii/S0167844213000815.

[7] D. Arnold and F. Brezzi. Locking-free finite element methods for shells. Mathematics of Computation of the American Mathematical Society, 66(217):1-14, 1997. ISSN 0025-5718, 1088-6842. doi: 10.1090/S0025-5718-97-00785-0. URL http://www .ams .org/mcom/1997-66-217/S0025-5718-97-00785-0/.

[8] D. N Arnold and R. S Falk. Asymptotic analysis of the boundary layer for the Reissner-Mindlin plate model. SIAM Journal on Mathematical Analysis, 27(2):486-514, 1996.

[9] M. Arroyo and T. Belytschko. Finite crystal elasticity of carbon nanotubes based on the exponential CauchyBorn rule. Physical Review B, 69(11):115415, March 2004. doi: 10.1103/PhysRevB.69.115415. URL https: //link.aps.org/doi/10.1103/PhysRevB.69.115415.

[10] B. Audoly and Y. Pomeau. Elasticity and Geometry. From hair curls to the non-linear response of shells. Oxford University Press, 2010.

[11] S. Balay, S. Abhyankar, M. F. Adams, Jed Brown, Peter Brune, Kris Buschelman, Lisandro Dalcin, Victor Eijkhout, William D. Gropp, Dinesh Kaushik, Matthew G. Knepley, Lois Curfman McInnes, Karl Rupp, Barry F. Smith, Stefano Zampini, Hong Zhang, and Hong Zhang. PETSc Users Manual. Technical Report ANL-95/11 Revision 3.7, Argonne National Laboratory, 2016. URL http://www.mcs.anl.gov/petsc.

[12] K. J. Bathe. Finite Element Procedures. 2 edition, 2014. ISBN 978-0979004957. 
[13] K. J Bathe and E. N Dvorkin. A four-node plate bending element based on Mindlin/Reissner plate theory and a mixed interpolation. International Journal for Numerical Methods in Engineering, 21(2):367-383, 1985.

[14] K-J. Bathe, F. Brezzi, and S. W. Cho. The MITC7 and MITC9 Plate bending elements. Computers 83 Structures, 32(3-4):797-814, 1989. ISSN 0045-7949. doi: 10.1016/0045-7949(89)90365-9. URL http://www. sciencedirect. com/science/article/B6V28-47XCOM5-GM/2/f5312a3ba4d9279fb60c8ed5bca5d954.

[15] K-J. Bathe, A. Iosilevich, and D. Chapelle. An evaluation of the mitc shell elements. Computers EJ Structures, $75(1): 1-30,2000$.

[16] L. Beirão da Veiga, C. Chinosi, C. Lovadina, and R. Stenberg. A-priori and a-posteriori error analysis for a family of Reissner-Mindlin plate elements. BIT Numerical Mathematics, 48(2):189-213, June 2008. ISSN 0006-3835, 1572-9125. doi: 10.1007/s10543-008-0175-y. URL http://link. springer.com/10.1007/s10543-008-0175-y.

[17] D.J. Benson, Y. Bazilevs, M.C. Hsu, and T.J.R. Hughes. Isogeometric shell analysis: The Reissner-Mindlin shell. Computer Methods in Applied Mechanics and Engineering, 199(5-8):276-289, January 2010. ISSN 0045-7825. doi: 10.1016/j.cma.2009.05.011. URL http://www.sciencedirect.com/science/article/B6V29-4WC1126-1/ 2/b073c14a2534022b5eda8399974d6164.

[18] P. Betsch, A. Menzel, and E. Stein. On the parametrization of finite rotations in computational mechanics: A classification of concepts with application to smooth shells. Computer Methods in Applied Mechanics and Engineering, 155(3):273 - 305, 1998. ISSN 0045-7825. doi: https://doi.org/10.1016/S0045-7825(97)00158-8. URL http://www.sciencedirect.com/science/article/pii/S0045782597001588.

[19] M. Bischoff and E. Ramm. Shear deformable shell elements for large strains and rotations. International Journal for Numerical Methods in Engineering, 40(23):4427-4449, December 1997. ISSN 1097-0207. doi: 10.1002/(SICI)1097-0207(19971215)40:23〈4427::AID-NME268〉3.0.CO;2-9. URL http://onlinelibrary . wiley . com/doi/10.1002/(SICI) 1097-0207(19971215) 40:23<4427: :AID-NME268>3.0.C0;2-9/abstract.

[20] K-U. Bletzinger, M. Bischoff, and E. Ramm. A unified approach for shear-locking-free triangular and rectangular shell finite elements. Computers \& Structures, 75(3):321-334, April 2000. ISSN 0045-7949. doi: 16/S0045-7949(99) 00140-6. URL http://www.sciencedirect.com/science/article/pii/S0045794999001406.

[21] D. Boffi and C. Lovadina. Analysis of new augmented Lagrangian formulations for mixed finite element schemes. Numerische Mathematik, 75(4):405-419, 1997.

[22] B. Bourdin, G. A. Francfort, and J. Marigo. The variational approach to fracture. Journal of Elasticity, 91(1): 5-148, 2008.

[23] F. Brezzi, J. Douglas, and L. D. Marini. Two families of mixed finite elements for second order elliptic problems. Numerische Mathematik, 47(2):217-235, June 1985. ISSN 0029-599X, 0945-3245. doi: 10.1007/BF01389710. URL https://link. springer. com/article/10.1007/BF01389710.

[24] C. Carstensen and J. Hu. A Posteriori Error Analysis for Conforming Mitc Elements for Reissner-Mindlin Plates. Mathematics of Computation, 77(262):611-632, 2008. ISSN 0025-5718. URL http://www.jstor.org/stable/ 40234526.

[25] D. Chapelle and K-J. Bathe. The Finite Element Analysis of Shells - Fundamentals. Springer, 2nd edition. edition, December 2010. ISBN 3-642-16407-2.

[26] D. Chapelle, D. L. Oliveira, and M. L. Bucalem. MITC elements for a classical shell model. Computers \&6 Structures, 81(8-11):523-533, May 2003. ISSN 0045-7949. doi: 10.1016/S0045-7949(02)00408-X. URL http: //www.sciencedirect.com/science/article/B6V28-47RJG6P-8/2/7efbc5c5c10fd7ec5a3bf 2eb8b557489.

[27] C. Chinosi and C. Lovadina. Numerical analysis of some mixed finite element methods for Reissner-Mindlin plates. Computational Mechanics, 16:36-44, April 1995. ISSN 0178-7675, 1432-0924. doi: 10.1007/s004660050046. URL http://www. springerlink. com/content/2v8n92dg407waue3/.

[28] P. G. Ciarlet. Theory of Shells. Elsevier, May 2000. ISBN 978-0-08-051123-8. Google-Books-ID: EYAxQ77o_6QC.

[29] F. Cirak, M. Ortiz, and P. Schröder. Subdivision surfaces: a new paradigm for thin-shell finite-element analysis. International Journal for Numerical Methods in Engineering, 47(12):2039-2072, 2000. ISSN 1097-0207. doi: 10.1002/(SICI)1097-0207(20000430)47:12〈2039::AID-NME872〉3.0.CO;2-1. URL http://dx.doi.org/10.1002/ (SICI) 1097-0207 (20000430) 47:12<2039: : AID-NME872>3.0.CO;2-1.

[30] S. Conti and F. Maggi. Confining thin elastic sheets and folding paper. Archive for Rational Mechanics and Analysis, 187(1):1-48, 2008.

[31] Electricité de France. Finite element code_aster, analysis of structures and thermomechanics for studies and research. Open source on www.code-aster.org, 1989-2017.

[32] R. Durán and E. Liberman. On mixed finite element methods for the Reissner-Mindlin plate model. Mathematics of Computation, 58(198):561-573, 1992. ISSN 0025-5718, 1088-6842. doi: 10.1090/S0025-5718-1992-1106965-0. URL http://www.ams.org/mcom/1992-58-198/S0025-5718-1992-1106965-0/.

[33] P. E. Farrell, Á. Birkisson, and S. W. Funke. Deflation techniques for finding distinct solutions of nonlinear partial 
differential equations. SIAM Journal on Scientific Computing, 37:A2026-A2045, 2015. doi: 10.1137/140984798.

[34] A. Foppl. Vorlesungen uber technische Mechanik. 1907.

[35] G. Gaël, J. Benoît, et al. Eigen v3. http://eigen.tuxfamily.org, 2010.

[36] J. S. Hale, M. Brunetti, S. Bordas, and C. Maurini. FEniCS-Shells. 2016. doi: 10.6084/m9.figshare.4291160.v1. URL https://figshare.com/articles/FEniCS-Shells/4291160.

[37] J. S. Hale, L. Li, C. N. Richardson, and G. N. Wells. Containers for Portable, Productive, and Performant Scientific Computing. Computing in Science \& Engineering, 19(6):40-50, November 2017. ISSN 1521-9615. doi: 10.1109/MCSE.2017.2421459. URL http://ieeexplore.ieee.org/document/7933304/.

[38] J.S. Hale. Meshless methods for shear-deformable beams and plates based on mixed weak forms. PhD thesis, Imperial College London, April 2013. URL http://spiral.imperial.ac.uk/handle/10044/1/14379.

[39] J.S. Hale and P.M. Baiz. A locking-free meshfree method for the simulation of shear-deformable plates based on a mixed variational formulation. Computer Methods in Applied Mechanics and Engineering, 241-244(0): 311-322, October 2012. ISSN 0045-7825. doi: 10.1016/j.cma.2012.06.010. URL http://www.sciencedirect. com/science/article/pii/S0045782512001983.

[40] W. Hamouche, C. Maurini, S. Vidoli, and A. Vincenti. Multi-parameter actuation of a neutrally stable shell: a flexible gear-less motor. Proceedings of the Royal Society of London A: Mathematical, Physical and Engineering Sciences, 473(2204), 2017. ISSN 1364-5021. doi: 10.1098/rspa.2017.0364. URL http://rspa. royalsocietypublishing.org/content/473/2204/20170364.

[41] J-F. Hiller and K-J. Bathe. Measuring convergence of mixed finite element discretizations: an application to shell structures. Computers \& Structures, 81(8):639-654, 2003.

[42] A. Ibarra, B. Roman, and F. Melo. The tearing path in a thin anisotropic sheet from two pulling points: Wulff's view. Soft Matter, 12(27):5979-5985, 2016.

[43] H-M. Jeon, Y. Lee, P-S. Lee, and K-J. Bathe. The MITC3+ shell element in geometric nonlinear analysis. Computers \& Structures, 146:91-104, January 2015. ISSN 0045-7949. doi: 10.1016/j.compstruc.2014.09.004. URL http://www.sciencedirect.com/science/article/pii/S0045794914001977.

[44] P. Kere and M. Lyly. Reissner-mindlin-von kármán type plate model for nonlinear analysis of laminated composite structures. Composite structures, 71(3):289-292, 2005.

[45] J. Kiendl, K.-U. Bletzinger, J. Linhard, and R. Wüchner. Isogeometric shell analysis with Kirchhoff-Love elements. Computer Methods in Applied Mechanics and Engineering, 198(49-52):3902-3914, November 2009. ISSN 00457825. doi: 10.1016/j.cma.2009.08.013. URL http://linkinghub.elsevier.com/retrieve/pii/S0045782509002680.

[46] R. C. Kirby and A. Logg. A Compiler for Variational Forms. ACM Trans. Math. Softw., 32(3):417-444, September 2006. ISSN 0098-3500. doi: 10.1145/1163641.1163644. URL http://doi.acm.org/10.1145/1163641.1163644.

[47] G. R. Kirchhoff. Uber das gleichgewicht und die bewegung einer elastischen scheibe. 1850.

[48] Y. Klein, E. Efrati, and E. Sharon. Shaping of elastic sheets by prescription of non-Euclidean metrics. Science, 315:1116-1120, February 2007.

[49] W. T. Koiter. A consistent first approximation in the general theory of thin elastic shells. The theory of thin elastic shells, pages 12-33, 1959.

[50] Warner T Koiter. On the foundations of linear theory of thin elastic shells. 1. Proceedings of the Koninklijke Nederlandse Akademie van Wetenschappen Series B-Physical Sciences, 73(3):169, 1970.

[51] J. Korelc. Multi-language and Multi-environment Generation of Nonlinear Finite Element Codes. Engineering with Computers, 18(4):312-327, November 2002. ISSN 0177-0667, 1435-5663. doi: 10.1007/s003660200028. URL https://link. springer.com/article/10.1007/s003660200028.

[52] P-S. Lee and K-J. Bathe. On the asymptotic behavior of shell structures and the evaluation in finite element solutions. Computers \& structures, 80(3):235-255, 2002.

[53] M. Lewicka, L. Mahadevan, and M. R. Pakzad. Models for elastic shells with incompatible strains. Proceedings of the Royal Society A: Mathematical, Physical and Engineering Science, 470(2165), 2014. doi: 10.1098/rspa. 2013.0604. URL http://rspa.royalsocietypublishing.org/content/470/2165/20130604. abstract.

[54] A. Logg and G. N. Wells. DOLFIN: Automated Finite Element Computing. ACM Trans. Math. Softw., 37(2): 20:1-20:28, April 2010. ISSN 0098-3500. doi: 10.1145/1731022.1731030. URL http://doi.acm.org/10.1145/ 1731022.1731030 .

[55] A. Logg, K-A. Mardal, and G. Wells, editors. Automated Solution of Differential Equations by the Finite Element Method, volume 84 of Lecture Notes in Computational Science and Engineering. Springer-Verlag Berlin and Heidelberg, 1 edition, 2012. ISBN 978-3-642-23099-8. URL https://dx.doi.org/10.1007/978-3-642-23099-8.

[56] C. Lovadina. A New Class of Mixed Finite Element Methods for Reissner-Mindlin Plates. SIAM Journal on Numerical Analysis, 33(6):2457-2467, December 1996. ISSN 0036-1429. URL http://www.jstor.org/stable/ 2158480 . 
[57] A. E. H. Love. A treatise on the mathematical theory of elasticity. Cambridge University Press, 2013.

[58] N. Lu and D. Kim. Flexible and Stretchable Electronics Paving the Way for Soft Robotics. Soft Robotics, 1(1): 53-62, July 2013. ISSN 2169-5172. doi: 10.1089/soro.2013.0005. URL https://www.liebertpub.com/doi/abs/ 10.1089 /soro. 2013.0005 .

[59] E. H. Mansfield. Bending, buckling and curling of a heated thin plate. Proceedings of the Royal Society of London. Series A, Mathematical and Physical Sciences, 268(1334):316-327, 1962. ISSN 00804630. URL http://www. jstor.org/stable/2414149.

[60] E.H. Mansfield. The bending and stretching of plates. Cambridge University Press, Cambridge, UK, 1989.

[61] C. Mardare. On the derivation of nonlinear shell models from three-dimensional elasticity. Rev. Roumaine Maths. Pures Appl., 53:499-522, 2008.

[62] K. Marguerre. Zur theorie der gekrümmten platte grosser formänderung. In Proceedings of the 5th international congress for applied mechanics, pages 93-101, 1938.

[63] J. Marigo, C. Maurini, and K. Pham. An overview of the modelling of fracture by gradient damage models. Meccanica, 51(12):3107-3128, 2016.

[64] S. Micheletti and S. Perotto. Reliability and efficiency of an anisotropic Zienkiewicz-Zhu error estimator. Computer Methods in Applied Mechanics and Engineering, 195(9-12):799-835, 2006.

[65] R. D. Mindlin. Influence of rotatory inertia and shear on flexural motions of isotropic, elastic plates. J. appl. Mech., 18:31, 1951.

[66] P. M. Naghdi. Foundations of elastic shell theory. Technical report, California Univ Berkeley Inst of Engineering Research, 1962.

[67] P. M. Naghdi. The theory of shells and plates. In Linear Theories of Elasticity and Thermoelasticity, pages 425-640. Springer, 1973.

[68] J. C. Nedelec. Mixed finite elements in R3. Numerische Mathematik, 35(3):315-341, September 1980. ISSN 0029-599X. doi: 10.1007/BF01396415. URL http://www. springerlink. com/content/vv750g6576n9p820/.

[69] T. D. Nguyen and G. N. Wells. Geometrically nonlinear formulation for thin shells without rotation degrees of freedom. Computer Methods in Applied Mechanics and Engineering, 197(33):2778 - 2788, 2008. ISSN 0045-7825. doi: https://doi.org/10.1016/j.cma.2008.01.001. URL http://www.sciencedirect.com/science/article/pii/ S004578250800025X.

[70] V. P. Nguyen, C. Anitescu, S. P. A. Bordas, and T. Rabczuk. Isogeometric analysis: An overview and computer implementation aspects. Mathematics and Computers in Simulation, 117:89-116, November 2015. ISSN 0378-4754. doi: 10.1016/j.matcom.2015.05.008. URL http://www. sciencedirect.com/science/article/ pii/S0378475415001214.

[71] N. Nguyen-Thanh, T. Rabczuk, H. Nguyen-Xuan, and S. P. A. Bordas. A smoothed finite element method for shell analysis. Computer Methods in Applied Mechanics and Engineering, 198(2):165-177, December 2008. ISSN 0045-7825. doi: 10.1016/j.cma.2008.05.029. URL http://www.sciencedirect.com/science/article/ pii/S0045782508002090.

[72] J. Pommier and Y. Renard. Getfem ++ , an open source generic $C++$ library for finite element methods. 2005. URL http://getfem.org/.

[73] P. Råback, M. Malinen, J. Ruokolainen, A. Pursula, and T. Zwinger. Elmer models manual. CSC-IT Center for Science, Helsinki, Finland, 2013.

[74] P. A. Raviart and J. M. Thomas. A mixed finite element method for 2-nd order elliptic problems. In Ilio Galligani and Enrico Magenes, editors, Mathematical Aspects of Finite Element Methods, pages 292-315, Berlin, Heidelberg, 1977. Springer Berlin Heidelberg. ISBN 978-3-540-37158-8.

[75] E. Reissner. On bending of elastic plates. Quarterly of Applied Mathematics, 5(1):55-68, 1947.

[76] J. A. Rogers, T. Someya, and Y. Huang. Materials and Mechanics for Stretchable Electronics. Science, 327(5973):1603-1607, March 2010. ISSN 0036-8075, 1095-9203. doi: 10.1126/science.1182383. URL http: //science. sciencemag.org/content/327/5973/1603.

[77] M. E. Rognes, R. C. Kirby, and A. Logg. Efficient Assembly of $\$ \mathrm{H}(\backslash \operatorname{mathrm}\{\operatorname{div}\}) \$$ and $\$ \mathrm{H}(\backslash \operatorname{mathrm}\{\operatorname{curl}\}) \$$ Conforming Finite Elements. SIAM Journal on Scientific Computing, November 2009. doi: 10.1137/08073901X. URL http://epubs.siam.org/doi/abs/10.1137/08073901X.

[78] M. E. Rognes, D. A. Ham, C. J. Cotter, and A. T. T. McRae. Automating the solution of PDEs on the sphere and other manifolds in FEniCS 1.2. Geoscientific Model Development, 6(6):2099-2119, December 2013. ISSN 1991-9603. doi: 10.5194/gmd-6-2099-2013. URL http://www.geosci-model-dev.net/6/2099/2013/.

[79] B. Roman. Fracture path in brittle thin sheets: a unifying review on tearing. International Journal of Fracture, 182(2):209-237, 2013. ISSN 1573-2673. doi: 10.1007/s10704-013-9869-5. URL http://dx.doi.org/10.1007/ s10704-013-9869-5. 
[80] Karam S. and Arthur L. Homogenization of Heterogeneous Thin and Thick Plates. Wiley-Blackwell, 2015. ISBN 9781119005247. doi: 10.1002/9781119005247. URL https://onlinelibrary.wiley.com/doi/abs/10.1002/ 9781119005247.

[81] J. Sanchez-Hubert and E. Sanchez-Palencia. Coques élastiques minces: propriétés asymptotiques. Masson edition, 1997.

[82] E. Sanchez-Palencia, O. Millet, and F. Béchet. Singular Problems in Shell Theory: Computing and Asymptotics. Number 54 in Lecture Notes in Applied and Computational Mechanics. Springer-Verlag, 2010.

[83] K. A. Seffen and S. D. Guest. Prestressed morphing bistable and neutrally stable shells. Journal of Applied Mechanics, Transactions ASME, 78(1):011002-1 to 011002-6, 2011.

[84] K.A. Seffen. Morphing bistable orthotropic elliptical shallow shells. Proceedings of the Royal Society A: Mathematical, Physical and Engineering Sciences, 463:67-83, 2007.

[85] K.A. Seffen and C. Maurini. Growth and shape control of disks by bending and extension. Journal of the Mechanics and Physics of Solids, 61(1):190-204, 2013. doi: 10.1016/j.jmps.2012.08.003. URL http://www . scopus . com/inward/record. url?eid=2-s2 . 0-84867574320\&partnerID=40\&md5=0db3ce9b07bc5a4090db32d135a60165.

[86] J. C Simo and M. S Rifai. A class of mixed assumed strain methods and the method of incompatible modes. International Journal for Numerical Methods in Engineering, 29(8):1595-1638, June 1990. ISSN 1097-0207. doi: 10.1002/nme.1620290802. URL http://onlinelibrary.wiley.com/doi/10.1002/nme.1620290802/abstract.

[87] K. Y. Sze, X. H. Liu, and S. H. Lo. Popular benchmark problems for geometric nonlinear analysis of shells. Finite elements in analysis and design, 40(11):1551-1569, 2004.

[88] P. Vannucci, B. Cochelin, N. Damil, and M. Potier-Ferry. An asymptotic-numerical method to compute bifurcating branches. International Journal for Numerical Methods in Engineering, 41(8): 1365-1389, December 1998. doi: 10.1002/(SICI)1097-0207(19980430)41:8〈1365::AID-NME332〉3.0.CO; 2-Y. URL https://onlinelibrary.wiley.com/doi/abs/10.1002/\%28SICI\%291097-0207\%2819980430\%2941\% 3A8\%3C1365\%3A\%3AAID-NME332\%3E3.0.CO\%3B2-Y.

[89] T. Von Kármán. Festigkeitsprobleme im maschinenbau. Teubner, 1910.

[90] G. N. Wells and T. D. Nguyen. A c0 discontinuous galerkin formulation for kirchhoff plates. Computer Methods in Applied Mechanics and Engineering, 196(35):3370 - 3380, 2007. ISSN 0045-7825. doi: https://doi.org/10. 1016/j.cma.2007.03.008. URL http://www.sciencedirect.com/science/article/pii/S0045782507001429.

[91] G. Wilson, D. A. Aruliah, C. T. Brown, Neil P. Chue Hong, Matt Davis, Richard T. Guy, Steven H. D. Haddock, Kathryn D. Huff, Ian M. Mitchell, Mark D. Plumbley, Ben Waugh, Ethan P. White, and Paul Wilson. Best Practices for Scientific Computing. PLOS Biology, 12(1):e1001745, January 2014. ISSN 1545-7885. doi: 10.1371/journal.pbio.1001745. URL http://journals.plos.org/plosbiology/article?id=10.1371/journal. pbio. 1001745. 